\title{
Cycles of transcription and local translation consolidate Arc protein at dendritic sites
}

Sulagna Das ${ }^{\mathrm{a}^{*}}$, Pablo J. Lituma ${ }^{\mathrm{b}}$, Pablo E. Castillo ${ }^{\mathrm{b}, \mathrm{c}}$, Robert H. Singer ${ }^{\mathrm{a}^{*}}$

${ }^{a}$ Department of Anatomy and Structural Biology

${ }^{\mathrm{b}}$ Dominick P. Purpura Department of Neuroscience

${ }^{\circ}$ Department of Psychiatry and Behavioral Sciences

Albert Einstein College of Medicine, Bronx, NY 10461, USA

* Corresponding authors:

Sulagna Das (sulagna.das@einsteinmed.org)

Robert H. Singer (robert.singer@einsteinmed.org)

Main Figures: 5

Supplementary Figures: 6 


\begin{abstract}
:
Maintenance of long-term memory requires transcription and translation of activity-regulated genes. Many of these are immediate early genes (IEGs) with short-lived mRNAs and proteins, often decaying rapidly after stimulation. It remains unknown how an IEG with rapid mRNA and protein turnover can impact long-lasting changes at the synapses. Here, we imaged the long-term transcription and translation dynamics of an IEG, Arc, important for memory consolidation, after stimulation in individual neurons. We demonstrated that the gene underwent reactivation in the same neuron without an additional stimulus, often at the same allele. The cycling of Arc transcription required protein synthesis. Indeed, phases of Arc translation were coordinated with the transcription cycles. Arc mRNAs from later cycles preferentially localized at dendritic sites marked by previous Arc protein, thereby creating hubs of local protein enrichment, potentially maintained by cycles of dendritic translation. These results identify novel characteristics of an IEG and provide insights into how unstable proteins may be sustained over the long-time scale of memory consolidation.
\end{abstract}




\section{Introduction:}

The ability to learn new information and store it for periods ranging from hours to days and even a lifetime is one of the most remarkable features of the brain. Memory consolidation requires modifications of synaptic connectivity and stabilization of these changes (1). The molecular basis of long-term memory involves both transcription and translation $(2,3)$. A class of genes termed as immediate early genes (IEGs) have established roles in the conversion of short- to long-term memory (4-6). However, IEG mRNAs and proteins are characterized by short halflives $(7,8)$ that are inconsistent with the time frame of long-term memory. Therefore, we attempt to resolve this conundrum by imaging transcription and translation of an IEG beyond the immediate early stage.

The Activity Regulated Cytoskeletal Associated (Arc) gene is an essential IEG for long-term memory and implicated in several neurodegenerative and cognitive disorders $(9,10)$. Growing evidence indicates that $A r c$ is a unique multifunctional IEG possessing both synaptic and nuclear functions (11). Arc regulates different forms of plasticity: long term potentiation (LTP), long term depression (LTD), and homeostatic plasticity $(5,12-14)$. The prevailing view of Arc regulation is mostly derived from studying a short temporal window after neuronal stimulation and behavioral tasks (15-17), although studies have shown that Arc proteins may persist longer $(11,14,18)$. Given the rapid molecular turnover of Arc (mRNAs and protein half-lives approximately $60 \mathrm{~min})(8,19,20)$, how their levels are sustained to mediate long-lasting changes at the synapses is unknown. To address this question, we performed high resolution imaging of Arc mRNA and proteins in hippocampal neurons over several hours after a brief stimulus. A knock-in Arc-PBS mouse, where the endogenous Arc gene was tagged with stem loops and detected by a fluorescent binding protein (20), allowed us to follow Arc mRNAs from synthesis to transport and decay with high spatial and temporal resolution. The tagged Arc behaves similarly to the untagged WT gene in transcriptional output, mRNA half-lives and protein production, as well as in spatial memory tasks (20).

We identified a novel feature of Arc gene regulation that help to reconcile the inconsistencies between its mRNA/protein half-lives and critical functions of Arc during long term memory storage. Transcription was reactivated beyond the immediate early (IE) phase without additional neuronal stimulation, creating a supply chain of mRNAs, which are then available to undergo 
translation in the dendrites. Indeed, cycles of transcription were coordinated with distinct phases of local Arc protein synthesis. Importantly, translation occurred in "hotspots" along the dendrites which facilitated the formation of local hubs of Arc protein. Over time, these hubs were maintained by subsequent cycles of mRNA localization and translation. The findings from this study provide a molecular mechanism by which unstable mRNAs with roles in synaptic plasticity could lead to long-lasting changes at the synapses required for memory consolidation.

\section{Results:}

\section{Arc gene undergoes transcriptional reactivation beyond the immediate early phase}

While most studies focused on the immediate early phase of Arc transcription, we investigated the long-term dynamics following a brief stimulation. Real-time imaging of transcription in hippocampal neurons from Arc-PBS mice was performed for four hours after an initial stimulation triggered by tetrodotoxin withdrawal (TTX-w) (Fig 1A). Induction of Arc transcription within 60 min of stimulation was deemed immediate early (IE). The IE activation and shutdown was followed by a second transcriptionally active ("ON") state, indicating a biphasic nature of Arc transcription. Since transcription was re-initiated in the same neuron after a prolonged OFF-period, we refer to this as a "reactivation" (Fig 1B, C). Reactivation was considered when the OFF-period after the IE-phase was $\geq 30$ mins. The average intensity trace of transcribing alleles from multiple neurons revealed that the two cycles of transcription were separated by an OFF period of $72 \pm 9.3 \mathrm{~min}$ (Fig 1D). We classified the transcriptional dynamics beyond the IE-phase (100 mins post stimulation) into three categories- sustained, reactivated, and delayed (de novo) transcription. Transcription was considered "sustained" when any one of the alleles was active till 100 mins, and "delayed" when de novo onset occurred after the IE phase. Notably, a significant $65 \pm 2.4 \%$ of transcription observed in the second cycle was a result of reactivation (Fig 1E), with an average onset time of $154.3 \pm 4.2 \mathrm{~min}$ (avg $\pm \mathrm{SEM}$ ) (Fig 1F). A heat map of intensities from individual alleles in the neurons undergoing reactivation revealed that reactivation occurred often at the same allelic in the population (Suppl. Fig1A). By comparing two different stimulation conditions (TTX-w and chemical LTP), $~ 60 \%$ of the transcriptionally active neurons from the IE-phase underwent reactivation in either case (Suppl. Fig1B). 
The probability of allelic reactivation was determined, and a significantly higher frequency for both alleles was observed (62\% for both vs 38\% for single, p < 0.05, paired t-test) (Fig 1G-H). Reactivation was a result of an initial stimulation since stochastic transcriptional bursts from neurons undergoing basal network activity in the absence of TTX did not exhibit distinct phases (Suppl. Fig 1C). Moreover, transcription of $\beta$-actin, a constitutive housekeeping gene did not display obvious reactivation under TTX-w (Suppl. Fig 1D), suggesting that the cycling is genespecific with precise temporal regulation. Next, the output from the two transcriptional cycles were characterized, and a significant reduction in nascent mRNA yield during reactivation compared to the IE phase for the same allele was observed (Suppl. Fig 2A, B). This likely resulted from a decrease in the duration of the transcriptional bursts in the reactivation phase (Suppl. Fig 2C), suggesting that the ON-state of the gene is shortened over time leading to a possible dampening of the cycles. Therefore, long-term imaging in individual neurons revealed the dynamics of Arc transcription beyond the IE phase and identified reactivation in a subset of neurons without any further stimulation.

\section{Optogenetic stimulation triggers transcriptional reactivation in cultures and in acute hippocampal slices}

The long-term transcriptional response of individual neurons to a specific stimulus strength was performed using optical stimulation of single cells by expressing channel rhodopsin ChR2 in the neurons from the Arc-PBS mouse and monitoring of transcription over time. Selective stimulation of the soma was performed (Fig 2A) with trains of $20 \mathrm{~Hz}$ pulses (25 pulses/train) separated by two minutes (Fig 2B). Measurements of nuclear $\mathrm{Ca}^{2+}$ used as a read-out for activity displayed robust increases post-stimulation (Fig 2C). After opto-stimulation, biphasic transcription was observed in individual neurons (Fig 2D). The reactivation probability was 54.2 $\pm 4.2 \%$, with average onset time of $146.4 \pm 7.1 \mathrm{~min}$ (Fig $\mathbf{2 E - F}$ ), comparable to that observed in global stimulation (TTX-w, cLTP). A similar decrease in the transcriptional output due to lower ON-duration in the reactivation phase compared to the IE phase was also observed (Suppl Fig 2D, E).

To visualize the long-term transcriptional behavior of the Arc gene in tissue, an optogenetic stimulation approach was developed for imaging real-time transcription in acute hippocampal slices from Arc PBS animals crossed with PCP-GFP transgenic mice. A cocktail of two viruses, 
AAVDJ-FLEX-ChIEF-tdTomato and AAV5-mCherry-Cre, was injected into the dentate gyrus to achieve Cre-specific expression of fast channel rhodopsin ChIEF and PCP-GFP in the granule cells (GC) (Fig 2G). A $25 \mathrm{~Hz}$ optical stimulation was delivered to the dentate gyrus (Fig $\mathbf{2 H}$ ), and transcription from both alleles in individual neurons was imaged for over four hours using two-photon microscopy (Fig 2I). In agreement with activity-dependent induction, neurons following optical stimulation showed an immediate early response of Arc transcription at $30 \mathrm{~min}$ followed by a decline and then a subsequent increase in transcribing neurons at $150 \mathrm{~min}(14.6 \pm$ $2.2 \%$ at $30 \mathrm{~min} ; 9.7 \pm 0.8 \%$ at $150 \mathrm{~min})(\mathbf{F i g} \mathbf{2 J})$. This second transcriptional phase primarily consisted of GCs undergoing reactivation and sustained transcription, and a small fraction of delayed de novo transcription (Fig 2K). Importantly, the time frame of reactivation (120-180 min) was similar to that observed in cultures, suggesting that the temporal regulation of Arc gene transcription was conserved in brain tissue. Hence, a better controlled (optical) method for triggering and imaging long-term Arc dynamics in cultures and tissue emphasized the generality of the biphasic transcriptional response.

\section{Reactivation occurs independently from increase in nuclear calcium}

Elevated levels of somatic and nuclear calcium have been implicated for induction of IEG transcription (17). Therefore, we investigated the dependency of transcription reactivation on $\mathrm{Ca}^{2+}$ activity. First, nuclear $\mathrm{Ca}^{2+}$ levels were measured using the indicator NLS-tagged jRGECO1a at different time points after stimulation (Suppl Fig 3A). An immediate increase in the frequency of $\mathrm{Ca}^{2+}$ transients (CaTs) at 10 min was observed, which peaked at $30 \mathrm{~min}(0.086$ $\pm 0.004 \mathrm{~Hz}$ ) and was maintained until $60 \mathrm{~min}$ (Suppl Fig 3B, C). This was followed by a significant reduction at $120 \mathrm{~min}(0.037 \pm 0.004 \mathrm{~Hz})$, and negligible activity was observed at 180 min (only 8 out of 40 cells exhibited CaTs). The amplitude of CaTs showed a similar profile: a rapid increase followed by a decrease at $120 \mathrm{~min}$ (Suppl Fig 3D). These findings showed a severe dampening of nuclear $\mathrm{Ca}^{2+}$ transients at the two-hour time point when reactivation was evident.

To confirm that reactivation occurred independently of $\mathrm{Ca}^{2+}$ rise, neuronal activity was silenced by reapplying TTX to the imaging media at $90 \mathrm{~min}$ (Fig 3A). Co-imaging of nuclear $\mathrm{Ca}^{2+}$ and Arc transcription was performed by expressing NLS- jRGECO1a and PCP-GFP in the same neuron $(\mathbf{F i g} 3 \mathbf{B}, \mathbf{C})$. Increase in nuclear $\mathrm{Ca}^{2+}$ levels and concomitant $A r c$ transcription was 
observed in the same neuron in the IE-phase. After reapplication of TTX (Fig 3B), CaTs were abolished. However, reactivation of Arc transcription was induced in those same neurons even though nuclear $\mathrm{Ca}^{2+}$ levels were undetectable (Fig 3C). Despite being lower than TTX-w, a notable $45 \pm 2.9 \%$ of neurons displayed reactivation after TTX reapplication (Fig 3D), with no significant difference in transcription onset times $(154.3 \pm 4.2$ for TTX-w versus $145.3 \pm 6.4$ for TTX-w + TTX, Fig 3E). Therefore, while the IE-phase was synchronous with elevated $\mathrm{Ca}^{2+}$ activity in the neuron, the reactivation onset appeared uncorrelated. This indicated that the later transcriptional phase was governed by a mechanism distinct from the conventional excitationtranscription coupling involving $\mathrm{Ca}^{2+}$ rise in the neurons.

\section{Transcriptional reactivation requires new protein synthesis}

Classically, IEGs including Arc, transcribe rapidly in response to stimulation, without the requirement for new protein synthesis $(21,22)$. We therefore assessed the role of de novo protein synthesis in the second phase of transcription. Translation was stalled using cycloheximide (CHX) at 90 min after the initial stimulation (Fig 3F). CHX addition severely impacted reactivation, only $7.8 \pm 2.1 \%$ activated neurons were transcribing two hours later (Fig 3G-I). Importantly, removal of CHX resulted in a robust induction of transcription (Fig 3G-I) with synchronous initiation times (median $=20 \mathrm{~min}$ after washout) (Fig 3J). To evaluate whether protein synthesis-dependent transcription occurred in tissue at later stages, acute hippocampal slices were briefly depolarized with $\mathrm{KCl}$, and maintained for two hours with or without $\mathrm{CHX}$, and fixed. In another set of slices, CHX was washed out and fixed (Suppl Fig 4A). Analysis of transcription sites (TS) in fixed slices after CHX treatment revealed that neurons transcribing at two hours were significantly reduced compared to no $\mathrm{CHX}$ and were rescued upon washout (Suppl Fig 4B, C). Moreover, the probability of transcription from both alleles was also restored (Suppl Fig 4D). Taken together, these findings indicate that the second phase of transcription requires de novo protein synthesis. We postulate that stalling translation elongation maintains a pool of readily translatable mRNA, which leads to a burst of protein synthesis upon CHX washout. These new proteins could then feedback to the nucleus to induce Arc gene transcription. 


\section{Arc mRNAs and proteins are maintained in the dendrites over the long term}

Given that at least two cycles of mRNA synthesis occurred, we assessed whether that led to corresponding changes in Arc mRNA levels in the dendrites. Time-lapse imaging of single mRNAs showed (Suppl Fig 5A, B) that the RNA numbers in dendrites was not constant but displayed fluctuations over time (Suppl Fig 5C). The RNA density plot in dendrites across multiple neurons displayed two phases - first one at 90 min followed by a plateau, and a second peak at 210 min (Suppl Fig 5D). Since the residence times of the Arc mRNAs in the dendrites was short (average 7.6 $\pm 1.2 \mathrm{~min}$ ) (Suppl Fig 5E), the increase in RNA density in the second phase was not a result of long-term persistence of these mRNAs, but primarily due to an additional supply which populate the dendrites over time.

Additional mRNAs trafficked and localized in the dendrites would result in local protein synthesis. To examine whether Arc proteins in the dendrites were being replenished, a reporter was designed to identify Arc proteins from the IE and late phases. The reporter driven by an activity-regulated ESARE promoter (23), contained a HaloTag upstream of the Arc coding sequence (CDS), followed by the 3'UTR comprising the cis-acting regulatory elements (Fig 4A). Cell permeable Halo-ligand conjugated to JF dyes (24) were used to label the Halo-tagged Arc proteins, similar to an approach used for $\beta$-actin (25). Spectrally distinct JF dyes- JF646 and JF549 dyes, detected Arc proteins synthesized from the IE and the second phase respectively (Suppl Fig 5F). Since a rapid phase of translation occurs at two hours immediately following transcription (26), the IE phase of protein synthesis was designated to be within three hours post stimulation. Labeling with JF646 till 150 min post stimulation displayed discrete puncta along the dendrites, corresponding to Arc proteins synthesized in the IE-phase (Suppl Fig 5G). A subsequent chase for 60 min with JF549 revealed a second phase of Arc synthesis, where the newly labeled proteins were in close proximity or overlapped with the previous 646 signal. The distances between the brightest JF646 puncta to the nearest JF549 signal was measured and majority $(75 \%)$ were within $3.4 \mu \mathrm{m}$, indicating the enrichment of Arc proteins from the first and second phases in discrete dendritic domains ("hubs") (Suppl Fig 5H). We propose that these hubs potentially represent regions where dendritic Arc levels are maintained over time. 


\section{Arc protein hubs are consolidated over time and serve as landing sites for Arc mRNAs from the second phase of transcription}

If the hubs were selectively consolidated compared to other dendritic regions, then repeated enrichment of Arc mRNAs and proteins should occur in these hubs. To test this possibility, the localization kinetics of mRNAs and proteins from the second phase was assessed relative to the hubs with high temporal resolution. A three-color real-time imaging approach was developed, where neurons from the Arc-PBS mouse were infected with two lentiviruses: one expressing PCP-GFP and the other expressing the Arc protein reporter (Fig 4A, B). The timeline of labeling and imaging has been depicted in the scheme (Fig 4C). A detailed analysis of JF646 signal revealed that Arc protein from the IE phase was not uniformly distributed but spatially restricted (Fig 4D, E). The local maxima of JF646 puncta intensity were used to mark a segment around the centroid of the peak ( $3 \mu \mathrm{m}$ on either side, based on Fig $5 \mathrm{H})$ to designate the hub marked by Arc protein from the IE phase (Fig 4F, G). Time lapse imaging with JF549 showed that Arc proteins translated from the second phase congregated in the region of the existing hub (Fig $\mathbf{4 H}$ ). However, these JF549 puncta were not long-lasting, suggesting possible degradation of the HaloArc protein. Importantly, the endogenous Arc mRNAs in the same dendrite also exhibited localization at or in the vicinity of the existing hubs, as shown by the kymographs (Fig 4I).

Quantification of the Arc protein from the second phase showed elevated levels specifically in the hub over time when compared with a neighboring dendritic segment (Fig 4J), supporting the idea that these hubs are being sustained over time by cycles of new protein synthesis. The peak of new Arc protein enrichment occurred at 252 mins after a latency period, indicating that the protein accumulation rates were biphasic. Notably, there was a spatial correlation between mRNA localization in the second phase with the protein hubs from the IE phase; a two-fold higher enrichment of Arc mRNAs in the initial hub versus the neighboring site was observed (Fig 4K). Once localized, the mRNAs persisted at these hubs as calculated from their residence times $(11.9 \pm 1.9 \mathrm{~min}$ in hub vs $6.3 \pm 1.1 \mathrm{~min}$ in neighboring site) (Fig $\mathbf{4 L})$. These results revealed distinct spatial and temporal features of dendritic Arc mRNAs and their cognate proteins: i) cycles of Arc protein synthesis separated by 2 hours, and ii) the emergence of local hubs of Arc proteins, which are reinforced over time, possibly by preferential localization of Arc mRNA from later cycles of transcription. 


\section{Long-term translation imaging reveals cycles of local Arc translation in dendrites}

To determine whether local translation at certain dendritic hotspots generated the Arc protein hubs, and the temporal regulation of the process, translation was imaged in real time with multimerized epitopes ("Suntag") on the Arc protein and detected with a genetically encoded single chain antibody ( $\mathrm{scFV}$ ) fused to a fluorescent protein (27-29). The Suntag-Arc reporter contained 24x repeats of the epitope in the N-terminus of Arc CDS with Arc mRNA 5' and 3'UTRs for translation regulation (Fig 5A). Fusing the 24x Suntag did not alter the localization of Arc protein (Suppl Fig 6A). The ability of the Suntag-Arc construct to report translating Arc mRNAs was tested by performing single molecule FISH (smFISH) for the mRNA and immunofluorescence (IF) against the Suntag protein in fixed neurons Fig 5B, left panel). Colocalization of smFISH spots with bright Suntag IF-signal showed mRNAs undergoing translation at two hours post stimulation. This was significantly reduced upon addition of Harringtonine (inhibitor of translation initiation) $(37.2 \pm 2.4 \%$ TTXw $2 \mathrm{~h}$ vs $16 \pm 2.3 \%$ Harringtonine, Fig 5C, D). Notably, the translating Arc mRNAs were maintained at 4 hour post stimulation, indicating that the mRNAs from both phases were translationally competent.

To investigate the spatio-temporal dynamics of local translation over time, Suntag-Arc reporter and scFV-GFP (Fig 5B, right panel) were co-expressed and imaged for several hours after stimulation (Fig 5E). Discrete foci, much brighter than the faster diffusing proteins were detected in the dendrites (Fig 5F), indicative of nascent sites of translation (TLS)(27). Tracking these TLS revealed that their numbers were not constant over time but displayed cycles of increase and decay (Fig 5G). Distribution of the OFF periods where no TLS are detected revealed a long $\mathrm{t}_{2}$ component of $55.4 \pm 8.3 \mathrm{~min}(\mathbf{F i g} \mathbf{5 H})$. These measurements closely mimicked the OFF periods of transcription ( 1hr), suggesting a temporal coordination of transcription and translation cycles. Spatial analysis of TLS distribution showed that they were not homogeneous but clustered along the dendrites (Fig 5F). This is representative of translation hotspots which are potential sites for elevated translation frequency (30). Accordingly, the appearance of multiple TLS ( 3 or more) in an $8 \mu \mathrm{m}$ dendritic segment within $120 \mathrm{~min}$ post stimulation was used as a criterion to define a translation hotspot (Fig 5I). A cumulative increase of TLS continued in these hotspots after IE-phase (150min), at a considerably higher rate than in a region without a hotspot (Fig 5J), closely resembling the patterns of spatially selective Arc protein enrichment 
(Fig 4). The abundance of nascent Arc proteins within the translation hotspots possibly facilitates the formation of local hubs.

The temporal kinetics of translation in these hotspots was obtained by binning the graph in Figure 5I into two 90 minute bins. The cumulative TLS counts in these two time bins revealed two distinct phases: the first reaching a plateau at $160 \mathrm{~min}$, followed by a second plateauing at 227 min (Suppl Fig 6B, C). A higher magnitude of increase, indicating more translation events, were detected for the first phase compared to the second phase, similar to the mRNA reactivation kinetics. Furthermore, by tracking the position and the integrated intensity of individual TLS which appeared in the two phases, translation was seen to occur in bursts lasting an average of $\sim 8 \mathrm{~min} /$ burst irrespective of the phase (Fig 5K). Hence, intermittent bursts of protein synthesis at local translation hotspots is a probable mechanism by which nascent Arc proteins are organized in the hubs and maintained over time (Fig 5L).

\section{Discussion}

In this study, the long-term dynamics of the Arc gene from transcription to translation in live neurons were followed using high-resolution imaging. This identified a novel temporal regulation of $A r c$, an IEG with critical roles in long term memory. Cycles of transcription and local translation demonstrated that intermittent phases of mRNA and protein synthesis maintain dendritic Arc protein levels over time. The protein is organized as local hubs, possibly as a result of local translation in certain hotspots and consolidated over time by subsequent translation at the same hotspot. This dynamics beyond the IE-phase provide a potential molecular basis by which a transient synaptic protein like Arc impacts long term spine remodeling and in turn memory.

The molecular events that occur inside the neuron during memory formation including the stabilization of synaptic contacts, have not been elucidated completely. It has been apparent that mRNA localization to activated spines plays a role in this process, and this leads to localized translation of specific proteins necessary for the structural and functional integrity of the postsynaptic structures $(3,6,31-34)$. To support memory consolidation, the activity-driven changes in the synapses must be sustained. For a structural protein such as $\beta$-actin, its mRNA is constitutively expressed, abundant, long-lived and can persist at, or revisit sites of activity to 
translate and promote stability of the cytoskeletal architecture within the postsynaptic region (25, 35). The plasticity protein, Arc, is synthesized from mRNAs transcribed upon activity, after they traffic to sites of activity to be translated locally (36-38). However, in contrast to actin, both Arc mRNAs and proteins are transient and degrade within a couple of hours $(8,39)$. For synaptic structures and physiology to be maintained, Arc must be persistently concentrated there, and this likely occurs via a second mechanism: cycles of transcription and translation in response to an initial stimulation. Since Arc is important for long term memory $(5,9)$, this molecular mechanism of cyclical transcription that begets additional rounds of transcription and synthesis creates a constant feedback loop that presumably supports memory consolidation.

Sustained levels of Arc mRNAs and proteins have been observed in cultured neurons (23), in different areas of the hippocampus after spatial learning tasks and during long term memory (4042). However, it has been challenging to distinguish whether one or more transcription events were occurring in the same or different neurons. Using the Arc-PBS mouse and the new Arc translation reporters, we characterized the different phases of gene expression with unprecedented temporal resolution. Reactivation of transcription in the same neuron predominantly accounted for the transcriptional events beyond the IE phase in both cultures and in tissue (Figs 1,2) irrespective of the stimulation condition, suggesting a conserved intrinsic feature of the Arc gene. Since, additional depolarization is not a requirement (Fig 3), transcriptional reactivation may occur by i) signaling cascades activated during IE-phase that remain sustained over long periods, and/or, ii) transcription factors which are synthesized in the IE phase and do not require $\mathrm{Ca}^{2+}$-mediated activation. The dependency of reactivation on protein synthesis (Fig 3) provided evidence favoring the latter possibility. Indeed, several TFs capable of regulating Arc expression directly or indirectly are rapidly translated IEGs , such as zif268 (43), Npas4 (44). For example, zif268 regulates late but not immediate early Arc expression after a behavioral task (45). Therefore, the molecular regulation of the two transcriptional phases is distinct due to their differential requirement of $\mathrm{Ca}^{2+}$ and de novo protein synthesis.

The phenomenon of reactivation is not just restricted to transcription; local translation of Arc mRNAs in the dendrites also occurs with a periodicity of $\sim 2$ hours. Reactivation could have several benefits. First, it allows production of short bursts of mRNAs and proteins without saturating the system. This is important for Arc, the levels of which need to be strictly regulated 
for cognitive flexibility (46). Second, reactivation maintains the RNA levels and efficiently replenishes the Arc hubs over long term. Finally, reactivation of Arc expression in a subset of neurons could favor their recruitment to a neuronal assembly supporting the memory trace (1). Therefore, the reactivation signature could potentially identify neurons involved in circuit strengthening during memory consolidation. Future studies are required to uncover the key modulators of reactivation, and the persistence of these cycles during long term memory.

The formation and maintenance of the local Arc hubs by periodic translation in hotspots highlight that the consolidation of Arc protein along the dendrites is spatially selective. These hotspots may either indicate regions of elevated synaptic activity where remodeling of spines is persisting, and/or regions of increased ribosome density (47) corresponding to high rates of translation $(30)$ (reviewed in $(32,48)$. Such hotspots of increased ribosomes and nascent proteins are often correlated with the synapse density, and found to be prevalent with both local stimulation as well as global paradigms (47). Translation in the hotspots allows the nascent Arc protein to accumulate locally to form the hubs. These local high concentrations of Arc could potentially facilitate the generation of Arc capsids for intercellular communication (49).

In the last decades, studies have independently focused on the contribution of transcription and local translation to long-term memory $(1-3,33)$. However, little is known about how these two important cellular processes converge to regulate memory consolidation. We have shown that coupling between transcription and translation is maintained for the subsequent cycles several hours after stimulation, consistent with the timing of stabilization of activity-driven changes. Cyclical gene expression may be a feature of genes which are under strict temporal regulation, as seen in developmental genes $(50,51)$, and studies further characterizing the regulation of the Arc cycles are needed. The long-term dynamics of Arc gene expression may serve as a template for studying other IEGs involved in memory. Determining the ubiquitous nature of these transcription- translation cycles, and whether they are synchronized or sequential for specific IEGs is critical to advance our knowledge of brain functions. Improvements in technologies to image mRNAs and proteins in vivo will pave the way towards understanding the temporal coupling of transcription-translation after a learning task, and whether perturbations of these cycles affect consolidation and persistence of memory. 


\section{Materials and Methods:}

\section{Plasmids and viral transduction}

All lentiviral constructs were cloned into the phage-ubc-RIG lentiviral vector. For Arctranslation construct, 24X Suntag or GCN4 repeats were PCR-amplified from the SINAPs construct (27). The promoter was the minimal ESARE promoter previously described (23) and synthesized as a gene block along with the 5'UTR sequences. The Arc coding sequence was synthesized based on the sequence (NM_001276684.1), and the 3'UTR sequence was a kind gift from Xiaowei Zhuang (28). The 3'UTR sequence was inserted before the woodchuck hepatitis virus posttranscriptional regulatory element (WPRE) in the lentiviral backbone.

The Halo-Arc protein reporter was designed based off the Halo-Actin-reporter (25), where the $\beta$ actin coding sequence and $\beta$-actin 3'UTR were replaced by the Arc CDS and Arc 3'UTR respectively. For PBS coat protein, PCP, we used the synonymously transformed version fused to GFP stdPCP-stdGFP (20).

The construct for red-shifted nuclear calcium indicators, NLS-jRGECO1a were cloned into the p323 backbone. The sequence for jRGECO1a was obtained from Addgene \#61563, and PCR amplified with primers to add NLS to the N-terminus. Lentiviral particles were produced by transfecting the expression vector with accessory plasmids, ENV, REV, VSVG and GAG in HEK 293 T cells. Collected lentiviral particles were purified with lenti-X concentrator (Clontech, Mountain View, CA).

\section{Animals}

Two animal strains were used- the Arc PBS-KI line and the $\operatorname{Arc}^{\mathrm{P} / \mathrm{P}}$ x PCP-GFP line. The ArcPBS line has been previously published and have been maintained at homozygosity. The genotyping primers and the conditions have been described in an earlier study (20). The PCPGFP mice were generated by introducing a neocassette containing CAG-stop/flox-NLS-PCPGFP in the ROSA 26 locus, where PCP-GFP expression is Cre-inducible. The Arc-PBS mouse $\left(\mathrm{Arc}^{\mathrm{P} / \mathrm{P}}\right.$ ) were crossed with the PCP-GFP animals to generate the The $\mathrm{Arc}^{\mathrm{P} / \mathrm{P}} \mathrm{x}$ PCP-GFP line are genotyped using the following PCRs for these primer sets: R26 wt forward primer (5'CCAAAGTCGCTCTGAGTTGT-3'), and reverse primers, R26 wt (5'CCAGGTTAGCCTTTAAGCCT-3'), and CMV R1 (5'-CGGGCCATTTACCGTAAGTT-3'), yielding a 250bp product for the WT allele, and a 329 bp product for the PCP-GFP respectively. 
Arc PBS were genotyped to All animals are being maintained at homozygosity with routine genotyping at Transnetyx. All animals are being maintained according to IUCAC guidelines.

\section{Stereotaxic Surgery and Hippocampal slice preparation}

$\operatorname{Arc}^{\mathrm{P} / \mathrm{P}}$ x PCP-GFP mice at postnatal day 27 (P27) were anesthetized with oxygen-isoflurane flowing at $1.5 \mathrm{ml} / \mathrm{min}$ and positioned into a Kopf stereotaxic instrument. A beveled Hamilton syringe injected 1-1.5 $\mu \mathrm{l}$ of 1:2 mix of AAV5-CaMKII-mcherry-Cre/AAV-DJ-FLEX-ChIEFtdTomato virus at coordinates targeting the dentate gyrus $(-2.1 \mathrm{~mm} \mathrm{~A} / \mathrm{P}, 1.7 \mathrm{~mm} \mathrm{M} / \mathrm{L}, 2.5 \mathrm{~mm}$ D/V). Animals were sacrificed 5-weeks post-surgery using anesthesia (4\% Isoflurane) followed by decapitation, and slices were prepared for two-photon microscopy. No differentiation of sex was done. Briefly, the animals were perfused with $20 \mathrm{~mL}$ of cold N-Methyl-D-glucamine (NMDG) solution containing in (mM): $93 \mathrm{NMDG}, 2.5 \mathrm{KCl}, 1.25 \mathrm{NaH}_{2} \mathrm{PO}_{4}, 30 \mathrm{NaHCO}_{3}, 20$ HEPES, 25 glucose, 5 sodium ascorbate, 2 Thiourea, 3 sodium pyruvate, $10 \mathrm{MgCl}_{2}, 0.5 \mathrm{CaCl}_{2}$, maintained at pH 7.35. The hippocampi were isolated and cut (300 $\mu \mathrm{m}$ thick) using a VT1200s microslicer in cold NMDG solution. Acute hippocampal slices were placed in a chamber with artificial cerebral spinal fluid solution (ACSF) solution composed of $124 \mathrm{NaCl}, 2.5 \mathrm{KCl}, 26$ $\mathrm{NaHCO}_{3}, 1 \mathrm{NaH}_{2} \mathrm{PO}_{4}, 2.5 \mathrm{CaCl}_{2}, 1.3 \mathrm{MgSO}_{4}$ and 10 glucose (in mM), and incubated in a 33$34^{\circ} \mathrm{C}$ water bath. All solutions were equilibrated with $95 \% \mathrm{O} 2$ and $5 \% \mathrm{CO}_{2}$ (pH 7.4). Postsectioning, acute slices were allowed to recover at room temperature for at least 45 min prior to experiments.

\section{2-photon imaging in slices and optical stimulation}

An Ultima 2P laser scanning microscope (Bruker Corp.) equipped with an Insight Deep See laser (Spectra-Physics) tuned to $910-930 \mathrm{~nm}$ was used to image Arc transcription in dentate granule cells (GCs) with 512 x 512 pixel resolution using $4 \mathrm{~mW}$ laser power measured at the $60 \mathrm{X}$ objective (Nikon, 1.0 NA). GCs expressing the PCP-GFP coat protein were imaged at $1 \mathrm{X}$ magnification to detect at least one transcribing neuron, which was then chosen as the region of interest (ROI) for $2 \mathrm{X}$ magnification. A Z-stack of $25 \mu \mathrm{m}$ thickness with $0.5 \mu \mathrm{m}$ steps was taken to assess baseline Arc transcription signals before optical stimulation.

Acute slices showing optimal ChIEF-tdTomato reporter expression (at least 75\% of DG was fluorescent) were selected for optical stimulation and imaging. The Invitro Ultima $2 \mathrm{P}$ 
microscope (Bruker Corp.) contains a Coherent $473 \mathrm{~nm}$ laser path that delivered optical stimulation of 25 pulses at $25 \mathrm{~Hz}$ repeated 20 times every $5 \mathrm{~s}(8 \mathrm{~mW}, 2-4 \mathrm{~ms}$ pulse duration). The stimulation area was specifically defined using customized Mark Point software (Bruker Corp.) and was empirically determined based on at least one transcribing neuron in the field of view as described above. After stimulation, Z-stack images of $25 \mu \mathrm{m}$ thickness with $0.5 \mu \mathrm{m}$ steps were acquired every 15 min for 4-5 hour.

\section{Depolarization of Slices and imaging of fixed slices}

Arc-PBS X PCP-GFP animals injected with AAV5-CaMKII-mcherry, were sacrificed 3-weeks post injection and acute hippocampal slices were prepared. The slices were briefly depolarized with $90 \mathrm{mM} \mathrm{KCl}$ for 3 mins and returned to ACSF at room temperature (RT). After 90 mins, slices were grouped into three treatment conditions: first group in ACSF, second group was incubated with CHX (100mg/ml) for 1 hour, and in the third group, CHX was added for 1 hour followed by washout. The first two groups were fixed at 2.5 hours, and the group with CHXwashout was fixed 45 min later to allow complete washout. Fixation was done with 4\% PFA in PBS overnight, and washed thrice with PBS, and then mounted onto slides with Prolong Diamond (Invitrogen). Imaging was performed on a wide-field fluorescence microscope built around an IX-81 stand (Olympus) and illumination was with 488nm laser, captured on EMCCD camera (Andor, iXon3 DU-897E-CS0-\#BV). 300nm z-stacks were acquired, and max-projected to obtain the images of GCs.

\section{Primary Hippocampal Neurons and Stimulation paradigms}

Mouse hippocampi were isolated from Arc PBS or Arc-PBS x PCP-GFP animals at post-natal day 0 or $1(\mathrm{P} 0 / \mathrm{P} 1)$. The tissue was digested in $0.25 \%$ tryspin for $15 \mathrm{~min}$ at $37{ }^{\circ} \mathrm{C}$, triturated and plated onto Poly-D-lysine (Sigma) coated glass bottom Mattek dishes at a density of 75,000 cells/dish for live imaging and 60,000 cells/dish for fixed cell imaging. Primary neurons were cultured in Neurobasal A media supplemented with B-27, GlutaMax and primocin (InvivoGen). Viral transduction was usually done at or after DIV 7, and neurons were imaged between DIV 16-19.

The paradigm for TTX-washout was used as described before $(20,52)$. Neurons were treated overnight (14-16 hours) with TTX $(2 \mu \mathrm{M})$, followed by washes, and fresh Hibernate A media 
(Brainbits) was added. In another set of experiments, a chemical LTP paradigm was used (53). Briefly, neuronal cultures were incubated with $50 \mu \mathrm{M}$ APV (Tocris) for 12 hours, followed by induction with $200 \mu \mathrm{M}$ glycine (Sigma) and $100 \mu \mathrm{M}$ picrotoxin (Tocris) in $\mathrm{Mg}^{2+}$-free Hibernate A media for $5 \mathrm{~min}$. Cells were then washed twice and returned to Hibernate A media with calcium and magnesium.

\section{Imaging in cultured hippocampal neurons}

Hippocampal neurons were imaged in Hibernate A media (Brainbits) at $35^{\circ} \mathrm{C}$ in a humidified chamber. Time-lapse imaging of transcription and translation was performed on a fluorescence microscope built around an IX-81 stand (Olympus) as described before $(20,27)$. The following lasers were used for illumination: $491 \mathrm{~nm}$ laser (Calypso-25, Cobolt, San Jose, CA), $561 \mathrm{~nm}$ line (LASOS-561-50, Lasertechnik GmbH, Germany) and a $640 \mathrm{~nm}$ line (CUBE 640-40C, Coherent Inc, Santa Clara, CA) were combined, expanded and delivered through the back port. The power for all lasers were controlled by an acousto-optic tunable filter (AOTF) (AOTFnC-400.650-TN, AA Opto-electronic).

The lasers were reflected by a four-band excitation dichroic mirror (Di01-R405/488/561/635, Semrock) to a 150x 1.45 N.A. oil immersion objective (Olympus). For transcription imaging, a 60x 1.40 NA oil immersion objective was used. The fluorescence collected by the same objective, were recorded on an EMCCD camera (Andor, iXon3 DU-897E-CS0-\#BV). The emission filters (FF01-525/50 for green and FF01-605/64 (Semrock) for red respectively) were mounted on a motorized filter wheel (FW-1000, Applied Scientific Instrumentation) for fast switching between wavelengths. For stimulation of single ChR2-expressing neurons, a sizeadjustable pinhole was used in the excitation light path to restrict the illumination to an area of approximately $10 \mu \mathrm{m}$ in diameter and prevent cross-activation of neighboring cells.

The microscope is equipped with an automated XY-stage (ASI, MS2000-XY) and a piezo-Z stage (ASI) for fast z-stack acquisition. The microscope was controlled and imaging performed on the Metamorph platform. The ChR2 stimulation paradigm was automated with custom journals in Metamorph. Briefly, the $10 \mu \mathrm{m}$ illumination spot was recorded and the ChR2 expressing neuron was moved to that area, and 5 images were taken. Next, 2 trains of stimulation of that neuron was done using $491 \mathrm{~nm}$ laser at $20 \mathrm{~Hz}$ for 20 times at power density $\left(7 \mathrm{~mW} / \mathrm{mm}^{2}\right)$, and switched back to wide-field illumination without the pinhole in the light path. For most 
experiments, a total of $11 \mathrm{z}$-stacks with $400 \mathrm{~nm}$ distance between stacks were acquired. Arc mRNAs and translation sites were imaged as z-stacks with $300 \mathrm{~nm}$ step size. The stacks were zprojected and used for analysis. For nuclear calcium, imaging was performed at single z-plane with $50 \mathrm{~ms}$ exposure times at $1 \mathrm{~Hz}$ acquisition rate.

\section{Single molecule fluorescence in situ hybridization (smFISH) and immunofluorescence (IF)}

Hippocampal neurons plated at 60,000 cells/Mattek dish were transduced with Suntag-Arc translation reporter for 10 days were stimulated with TTX-washout paradigm at DIV 19 and fixed. The neurons were then permeabilized and smFISH-IF was performed according to the protocol described in (54). Briefly, 100nM probes against 24X Suntag sequence and primary antibodies against GCN4 epitopes (Clone C11L34, Ab00436-1.4, Absolute antibody, Wilton, UK) was used in hybridization buffer for 3 hours at $37^{\circ} \mathrm{C}$. After washes, the cells were incubated with Alexa Fluor 647 secondary antibody (Life Technologies) and mounted using ProLong diamond antifade reagent with DAPI (Life Technologies). Images were taken in a custom upright widefield Olympus BX-63 microscope equipped with a Lumencor SOLA-Light engine, ORCA-R2 Digital CCD camera (Hamamatsu), SuperApochromatic 60x/1.35 NA Olympus objective (UPLSAPO60XO) and zero pixel shift filter sets: DAPI-5060C-Zero, Cy3-4040C-Zero and Cy5-4040C-Zero (Semrock). Image pixel size: XY, $107.5 \mathrm{~nm}$; Z-steps, $200 \mathrm{~nm}$. The sequences for FISH probes have been described in (27).

\section{Image Analysis}

Fixed cell analysis: smFISH - IF images were analyzed using FISH Quant (55). Briefly, the FISH spots in the dendrites were filtered and fit to a 3D Gaussian to determine the coordinates of the mRNAs in Cy3 channel. The intensity and the width of the 3D Gaussian were thresholded to exclude non-specific and autofluorescent particles. Similarly, independent analysis of Suntag spots were performed using FISH-Quant. Co-localization analysis was done using by finding mRNAs which had Suntag signal within $300 \mathrm{~nm}$ distance.

\section{Live-cell imaging data analysis:}

Transcription site analysis: Time-lapse images of transcription were obtained after maximum intensity projection of the z-series. The transcription sites (TS) in time lapse images were tracked 
and their fluorescence intensities were quantified with custom programs written in Matlab (Mathworks). The intensity of TS was normalized to the diffusive PCP-GFP signal in the nucleus. Intensity traces were subjected to a rolling average of 3 frames to remove fast fluctuations in fluorescence signal. A value $\geq 1.4$ was considered $\mathrm{ON}$-state of the gene (20). Values below 1.4 were considered OFF-state. An OFF-period of at least 30 mins between two transcriptional bursts, when the second burst was after 90 min post stimulation was used as criterion to designate reactivation. If any of the alleles was transcriptionally active beyond the IE phase and continued till 100 min post stimulation, then the transcriptional state was considered sustained. Induction of de novo transcription after 100 min of stimulation is marked as an delayed (de novo) event. To determine the transcriptional bursting parameters in each cycle, the images were binned into two time segments: IE (15-75 $\mathrm{min})$ and reactivation phase (105-200 min). Each phase can be composed of multiple transcriptional bursts (56). The duration of the ON-state, and the area under each burst were quantified and the sum of all the bursts in each phase have been represented.

Translation site analysis: To track translation sites (TLS) in neurons, we chose dendritic segments which are $>30 \mu \mathrm{m}$ from soma or in secondary dendrites. Presence of at least one TLS at the start of imaging was used as a criterion for choosing a particular dendrite. Semi-automated tracking of TLS was performed using the custom-built program on MATLAB, with a gap of 2 frames allowed to be treated as the same site. TLS which lasted for at least 3 frames were used for analysis. The intensity of TLS was normalized to the diffusive scFv-sfGFP signal in the dendrites. A threshold of 1.5 was used as a cutoff to be considered as a translating spot. The gradual increase in the intensity of the translation spots indicates more nascent peptide synthesis and therefore deemed to be translating mRNA. The time between the active translation bursts across multiple TLS represented the OFF-durations, which were plotted as an inverse cumulative distribution function (1-CDF) and fitted to a 2-component exponential fit (goodness of fit test performed in MATLAB).

Single mRNA analysis: Single particle tracking of mRNAs was performed using Trackmate plugin on Fiji. The dendrites were straightened before analysis. Tracks shorter than 3 frames were not considered. mRNA counts were normalized to the length of the dendrites to determine 
RNA density. The track lengths of both stationary and moving mRNAs were used to calculate the residence times of mRNAs in dendrites. For Figure 5, kymographs were plotted for straightened dendrites. The number of mRNAs which last for $\geq 2$ frames ( $3 \mathrm{~min}$ ) were considered for mRNA counts in the pre-existing versus the neighboring sites.

Pulse-chase assay: The puncta from JF646 and JF549 channels were localized with Analyze particles plugin on Image J. The puncta were diffraction limited spots, which could be fitted to a 2D-Gaussian. High intensity diffusible 646 signal was not considered for analysis. The coordinates were noted. The distances were calculated from the brightest JF646 puncta using the nearest neighbor analysis. JF646 puncta whose brightness were above $20 \%$ above background fluorescence (diffusive signal in dendrites) was used for analysis. From the frequency distributions of the distances, 75 percentile corresponding to $3.4 \mu \mathrm{m}$ was used as the cutoff to designate protein enrichment in space. Accordingly, we used a segment of $6 \mu \mathrm{m}$ length $(3 \mu \mathrm{m}$ on either side of the centroid of the brightest 646 puncta) to define the Arc protein hub from IEphase.

Nuclear $\mathrm{Ca}^{2+}$ imaging analysis:_Images were acquired at $1 \mathrm{~Hz}$ frequency at single planes. The time series were used, and the ROIs for the nuclei were detected in a semi-automated manner in Fiji. The same cells were imaged over time and the same ROIs with required correction for $x-y$ drift were used to quantify fluorescence. The average value from the first 3 frames was treated as baseline fluorescence $(F)$, and the following change in fluorescence $(\Delta F)$ was measured. The values in the traces are represented as $\Delta \mathrm{F} / \mathrm{F}$. Traces were fitted to a peak-fitting algorithm and the maximum likelihood analysis was performed for peak assignment. The frequency and amplitude of the peaks were calculated.

\section{Transcription imaging in slices}

Images of GC nuclei were filtered, and then semi-automated detection of transcription sites was performed based on fitting to a 2D-Gaussian and ROIs of 28-32 pixels was used for each transcribing allele. The intensity of each transcription site was normalized by the background signal of the nucleus using the same ROI dimension. The normalized intensity value of 1 represents that no transcription sites are detected. An intensity threshold of $10 \%$ change (i.e. 
values 1.1 or higher) was used to designate a transcription site. The same transcription site was followed over time to measure the change in normalized intensity values, and below 1.1 was considered as transcriptional shutdown. Quantification of total transcribing cells were performed as follows:

$\%$ of total transcribing cells $=\left(\frac{\text { Total no. of cells with TS }}{\text { Total number of PCP-GFP positive cells }}\right) \times 100$

\section{Statistics}

One-way ANOVA (Dunett's multiple comparison) was used to determine statistical significance for more comparison between more than two groups. Student's t-test determined statistical significance for all other experimental conditions. Paired t-test was performed when comparing the same neuron or the same allele from the IE and reactivation phases. Normality test for all conditions was performed using the Shapiro-Wilk test. Wilcoxon Signed Ranks test was used for not normally distributed data. All statistical tests were performed on Graph Pad Prism.

Author Contributions: S.D. conceptualized the project, and with R.H.S. designed the research. S.D. performed real-time imaging of transcription, translation in cultures and analyzed all expeimental data. P.J.L. performed stereotaxic surgeries, and two-photon microscopy of transcription in slices. All authors discussed the results. S.D. and R.H.S wrote the first manuscript, which was edited by P.E.C. and P.J.L.

Acknowledgments: We are grateful to Xiuhua Meng and Melissa Lopez-Jones for technical assistance, and Chiso Nwokafor for animal maintenance. We thank all members of Robert H. Singer and Pablo E. Castillo laboratories for insightful discussions. We are grateful to Luke Lavis from Janelia Research Campus for providing the JF646 and JF549 dyes. We would like to thank Dong-woo Hwang and Weihan Li for critical reading of the manuscript.

The work was supported by NIH grants MH122961 to S.D; NS083085 to R.H.S.; MH125772, MH116673, NS115543, and NS113600 to P.E.C.; a Ruth L. Kirschstein NRSA Fellowship MH109267 to P.J.L. 


\section{References:}

1. A. Asok, F. Leroy, J. B. Rayman, E. R. Kandel, Molecular Mechanisms of the Memory Trace. Trends in neurosciences 42, 14-22 (2019).

2. C. M. Alberini, E. R. Kandel, The regulation of transcription in memory consolidation. Cold Spring Harbor perspectives in biology 7, a021741 (2014).

3. M. A. Sutton, E. M. Schuman, Dendritic protein synthesis, synaptic plasticity, and memory. Cell 127, 49-58 (2006).

4. W. Tischmeyer, R. Grimm, Activation of immediate early genes and memory formation. Cellular and molecular life sciences : CMLS 55, 564-574 (1999).

5. N. Plath et al., Arc/Arg3.1 is essential for the consolidation of synaptic plasticity and memories. Neuron 52, 437-444 (2006).

6. D. O. Wang et al., Synapse- and stimulus-specific local translation during long-term neuronal plasticity. Science 324, 1536-1540 (2009).

7. K. M. Tyssowski et al., Different Neuronal Activity Patterns Induce Different Gene Expression Programs. Neuron 98, 530-546 e511 (2018).

8. V. R. Rao et al., AMPA receptors regulate transcription of the plasticity-related immediate-early gene Arc. Nature neuroscience 9, 887-895 (2006).

9. C. R. Bramham et al., The Arc of synaptic memory. Experimental brain research 200, 125-140 (2010).

10. E. Korb, S. Finkbeiner, Arc in synaptic plasticity: from gene to behavior. Trends in neurosciences 34, 591-598 (2011).

11. C. R. Bramham, P. F. Worley, M. J. Moore, J. F. Guzowski, The immediate early gene arc/arg3.1: regulation, mechanisms, and function. The Journal of neuroscience : the official journal of the Society for Neuroscience 28, 11760-11767 (2008).

12. J. D. Shepherd et al., Arc/Arg3.1 mediates homeostatic synaptic scaling of AMPA receptors. Neuron 52, 475-484 (2006).

13. S. Chowdhury et al., Arc/Arg3.1 interacts with the endocytic machinery to regulate AMPA receptor trafficking. Neuron 52, 445-459 (2006).

14. E. Messaoudi et al., Sustained Arc/Arg3.1 synthesis controls long-term potentiation consolidation through regulation of local actin polymerization in the dentate gyrus in vivo. The Journal of neuroscience : the official journal of the Society for Neuroscience 27, 10445-10455 (2007).

15. J. F. Guzowski, B. L. McNaughton, C. A. Barnes, P. F. Worley, Environment-specific expression of the immediate-early gene Arc in hippocampal neuronal ensembles. Nature neuroscience $\mathbf{2}$, 1120-1124 (1999).

16. F. T. Gallo, C. Katche, J. F. Morici, J. H. Medina, N. V. Weisstaub, Immediate Early Genes, Memory and Psychiatric Disorders: Focus on c-Fos, Egr1 and Arc. Frontiers in behavioral neuroscience 12, 79 (2018).

17. E. L. Yap, M. E. Greenberg, Activity-Regulated Transcription: Bridging the Gap between Neural Activity and Behavior. Neuron 100, 330-348 (2018).

18. V. Ramirez-Amaya et al., Spatial exploration-induced Arc mRNA and protein expression: evidence for selective, network-specific reactivation. The Journal of neuroscience : the official journal of the Society for Neuroscience 25, 1761-1768 (2005).

19. J. Soule et al., Balancing Arc synthesis, mRNA decay, and proteasomal degradation: maximal protein expression triggered by rapid eye movement sleep-like bursts of muscarinic cholinergic receptor stimulation. The Journal of biological chemistry 287, 22354-22366 (2012).

20. S. Das, H. C. Moon, R. H. Singer, H. Y. Park, A transgenic mouse for imaging activity-dependent dynamics of endogenous Arc mRNA in live neurons. Science advances 4, eaar3448 (2018).

21. M. E. Greenberg, E. B. Ziff, Stimulation of 3 T3 cells induces transcription of the c-fos protooncogene. Nature 311, 433-438 (1984). 
22. R. N. Saha, S. M. Dudek, Splitting hares and tortoises: a classification of neuronal immediate early gene transcription based on poised RNA polymerase II. Neuroscience 247, 175-181 (2013).

23. T. Kawashima et al., Synaptic activity-responsive element in the Arc/Arg3.1 promoter essential for synapse-to-nucleus signaling in activated neurons. Proceedings of the National Academy of Sciences of the United States of America 106, 316-321 (2009).

24. J. B. Grimm et al., A general method to fine-tune fluorophores for live-cell and in vivo imaging. Nature methods 14, 987-994 (2017).

25. Y. J. Yoon et al., Glutamate-induced RNA localization and translation in neurons. Proceedings of the National Academy of Sciences of the United States of America 113, E6877-E6886 (2016).

26. J. D. Shepherd, M. F. Bear, New views of Arc, a master regulator of synaptic plasticity. Nature neuroscience 14, 279-284 (2011).

27. B. Wu, C. Eliscovich, Y. J. Yoon, R. H. Singer, Translation dynamics of single mRNAs in live cells and neurons. Science 352, 1430-1435 (2016).

28. C. Wang, B. Han, R. Zhou, X. Zhuang, Real-Time Imaging of Translation on Single mRNA Transcripts in Live Cells. Cell 165, 990-1001 (2016).

29. M. E. Tanenbaum, L. A. Gilbert, L. S. Qi, J. S. Weissman, R. D. Vale, A protein-tagging system for signal amplification in gene expression and fluorescence imaging. Cell 159, 635-646 (2014).

30. C. Job, J. Eberwine, Identification of sites for exponential translation in living dendrites. Proceedings of the National Academy of Sciences of the United States of America 98, 1303713042 (2001).

31. C. E. Holt, K. C. Martin, E. M. Schuman, Local translation in neurons: visualization and function. Nat Struct Mol Biol 26, 557-566 (2019).

32. S. Das, M. Vera, V. Gandin, R. H. Singer, E. Tutucci, Intracellular mRNA transport and localized translation. Nat Rev Mol Cell Biol 22, 483-504 (2021).

33. M. Costa-Mattioli, W. S. Sossin, E. Klann, N. Sonenberg, Translational control of long-lasting synaptic plasticity and memory. Neuron 61, 10-26 (2009).

34. R. J. Kelleher, 3rd, A. Govindarajan, S. Tonegawa, Translational regulatory mechanisms in persistent forms of synaptic plasticity. Neuron 44, 59-73 (2004).

35. A. R. Buxbaum, Y. J. Yoon, R. H. Singer, H. Y. Park, Single-molecule insights into mRNA dynamics in neurons. Trends in cell biology 25, 468-475 (2015).

36. O. Steward, C. S. Wallace, G. L. Lyford, P. F. Worley, Synaptic activation causes the mRNA for the IEG Arc to localize selectively near activated postsynaptic sites on dendrites. Neuron 21, 741 751 (1998).

37. O. Steward, S. Farris, P. S. Pirbhoy, J. Darnell, S. J. Driesche, Localization and local translation of Arc/Arg3.1 mRNA at synapses: some observations and paradoxes. Front Mol Neurosci 7, 101 (2014).

38. Y. Na et al., Real-Time Imaging Reveals Properties of Glutamate-Induced Arc/Arg 3.1 Translation in Neuronal Dendrites. Neuron 91, 561-573 (2016).

39. S. Farris, G. Lewandowski, C. D. Cox, O. Steward, Selective localization of arc mRNA in dendrites involves activity- and translation-dependent mRNA degradation. The Journal of neuroscience : the official journal of the Society for Neuroscience 34, 4481-4493 (2014).

40. V. Ramirez-Amaya, A. Angulo-Perkins, M. K. Chawla, C. A. Barnes, S. Rosi, Sustained transcription of the immediate early gene Arc in the dentate gyrus after spatial exploration. The Journal of neuroscience : the official journal of the Society for Neuroscience 33, 1631-1639 (2013).

41. L. M. Igaz, M. R. Vianna, J. H. Medina, I. Izquierdo, Two time periods of hippocampal mRNA synthesis are required for memory consolidation of fear-motivated learning. The Journal of neuroscience : the official journal of the Society for Neuroscience 22, 6781-6789 (2002).

42. D. Nakayama et al., Long-delayed expression of the immediate early gene Arc/Arg3.1 refines neuronal circuits to perpetuate fear memory. The Journal of neuroscience : the official journal of the Society for Neuroscience 35, 819-830 (2015). 
43. L. Li, J. Carter, X. Gao, J. Whitehead, W. G. Tourtellotte, The neuroplasticity-associated arc gene is a direct transcriptional target of early growth response (Egr) transcription factors. Molecular and cellular biology 25, 10286-10300 (2005).

44. X. Sun, Y. Lin, Npas4: Linking Neuronal Activity to Memory. Trends in neurosciences 39, 264275 (2016).

45. Z. Penke, C. Chagneau, S. Laroche, Contribution of Egr1/zif268 to Activity-Dependent Arc/Arg3.1 Transcription in the Dentate Gyrus and Area CA1 of the Hippocampus. Frontiers in behavioral neuroscience 5, 48 (2011).

46. M. J. Wall et al., The Temporal Dynamics of Arc Expression Regulate Cognitive Flexibility. Neuron 98, 1124-1132 e1127 (2018).

47. C. Sun et al., The prevalence and specificity of local protein synthesis during neuronal synaptic plasticity. Science advances 7, eabj0790 (2021).

48. C. Job, J. Eberwine, Localization and translation of mRNA in dendrites and axons. Nature reviews. Neuroscience 2, 889-898 (2001).

49. E. D. Pastuzyn et al., The Neuronal Gene Arc Encodes a Repurposed Retrotransposon Gag Protein that Mediates Intercellular RNA Transfer. Cell 172, 275-288 e218 (2018).

50. D. Kim, D. Grun, A. van Oudenaarden, Dampening of expression oscillations by synchronous regulation of a microRNA and its target. Nat Genet 45, 1337-1344 (2013).

51. G. J. Hendriks, D. Gaidatzis, F. Aeschimann, H. Grosshans, Extensive oscillatory gene expression during C. elegans larval development. Mol Cell 53, 380-392 (2014).

52. R. N. Saha et al., Rapid activity-induced transcription of Arc and other IEGs relies on poised RNA polymerase II. Nature neuroscience 14, 848-856 (2011).

53. P. G. Donlin-Asp, C. Polisseni, R. Klimek, A. Heckel, E. M. Schuman, Differential regulation of local mRNA dynamics and translation following long-term potentiation and depression. Proceedings of the National Academy of Sciences of the United States of America 118, (2021).

54. C. Eliscovich, S. M. Shenoy, R. H. Singer, Imaging mRNA and protein interactions within neurons. Proceedings of the National Academy of Sciences of the United States of America 114, E1875-E1884 (2017).

55. F. Mueller et al., FISH-quant: automatic counting of transcripts in 3D FISH images. Nature methods 10, 277-278 (2013).

56. A. J. M. Larsson et al., Genomic encoding of transcriptional burst kinetics. Nature, (2019). 


\section{Figure Legends:}

Figure 1: Long-term imaging of Arc transcription after stimulation. (A) Schematic of the stimulation paradigm, and the imaging time course. (B) Representative images showing the PCP-GFP labeled nucleus of a single neuron. Yellow arrows indicate transcribing Arc alleles. (C) Intensity trace of the transcribing allele in B. Solid line shows normalization to nuclear background. Dashed line indicates threshold for inclusion criteria as active transcription. (D) Average intensity trace from individual alleles of multiple neurons. Shaded areas indicate transcriptional activity above threshold $(n=58$ neurons from 6 independent experiments). (E) Graph indicates the percentage of cells displaying different transcriptional states in the second phase (post $90 \mathrm{~min}$ ). Reactivation is considered when the OFF-periods are $\geq 30 \mathrm{~min}$ between two transcription events, when the second one is $100 \mathrm{~min}$ post stimulation. ( $\mathrm{n}=92$ neurons, 7 independent experiments, each circle represents one experiment). (F) Frequency distribution of reactivation onset times ( $\mathrm{n}=45$ neurons, 6 independent experiments). (G) Representative images showing reactivation of both alleles albeit with different onset times. Arrows indicate transcribing alleles, yellow indicates first allele, and blue points to the second allele. (H) Frequency of reactivation from both alleles or from a single $\operatorname{Arc}$ allele ( $\mathrm{n}=45$ neurons, 6 independent experiments, each circle represents one experiment). Scale bar is 5 microns. Error bars indicate SEM.

Figure 2: Reactivation of Arc transcription after optical stimulation in cultures and in tissue. (A) Hippocampal neurons from Arc-PBS animals were infected with lentiviruses expressing ChR2-mCherry. The soma of mCherry-positive neurons were stimulated with trains of $488 \mathrm{~nm}$ light using a pinhole (blue circle). (B) Stimulation paradigm for triggering activity. (C) Nuclear $\mathrm{Ca}^{2+}$ measurements under basal conditions and after optical stimulation. (D) Representative images showing the PCP-GFP labeled nucleus of a single neuron after optical stimulation. Yellow arrows show transcribing Arc alleles. Scale bar $10 \mu \mathrm{m}$. (E) Percentage of transcribing cells displaying different transcriptional states in later stages (90 min after optical stimulation) ( $\mathrm{n}=27$ neurons from 4 independent experiments). (F) Frequency distribution of reactivation onset times after optical stimulation of ChR2-expressing neurons. (G) Approach for Cre-dependent expression of PCP-GFP and ChIEF in the granule cells by injecting a cocktail of two viruses- CaMKII $\alpha$-Cre and DJ-FLEX-ChIEF to the dentate gyrus of Arc-PBS x PCP-GFP mice. (H) Stimulation paradigm for ChIEF (473nm) and two-photon imaging of GCs (910 nm illumination). (I) Representative images of two GC nuclei displaying transcription after optical stimulation. Orange outline shows neuron with transcriptional reactivation, while the one with green outline has sustained activation. Scale bar $10 \mu \mathrm{m}$. (J) Total percentage of transcribing GC neurons after optical stimulation revealed two cycles $(14.6 \pm 2.2 \%$ at $30 \mathrm{~min}, 9.7 \pm 0.74 \%$ at $150 \mathrm{~min}, * * * \mathrm{p}=0.003$ at 
$30 \mathrm{~min},{ }^{*} \mathrm{p}=0.01$ at $150 \mathrm{~min},{ }^{*} \mathrm{p}=0.04$ at $180 \mathrm{~min}$, compared to baseline $0 \mathrm{~min}$, one-way ANOVA). (K) Distribution of the different transcriptional states in GC nuclei in the second phase (100min post stimulation). $\mathrm{n}=5$ slices, 5 animals (J, K). Error bars indicate SEM. *** denotes $\mathrm{p}<0.005$, * denotes $\mathrm{p}<$ 0.05 .

\section{Figure 3: Reactivation of $\mathrm{Arc}$ transcription is independent of $\mathrm{Ca}^{2+}$ rise but requires protein} synthesis. (A) Schematic of stimulation paradigm. TTX was reapplied at $90 \mathrm{~min}$ to test the effect on reactivation. (B-C) Neurons co-expressing NLS-jRGECO1a and PCP-GFP were imaged for nuclear $\mathrm{Ca}^{2+}$ levels and Arc transcription. Increase in nuclear $\mathrm{Ca}^{2+}$ transients at $15 \mathrm{~min}$ of TTX-w continued to $60 \mathrm{~min}$, and ceased after reapplying TTX in B. Imaging TS in the same neuron showed reactivation after TTX addition in C. (D) Different transcriptional states after TTX reapplication in neurons activated in IE-phase ( $\mathrm{n}=43$ neurons from 3 independent experiments). (E) Comparison of reactivation onset times ( $\mathrm{n}=20$ neurons for TTX-w + TTX; 45 neurons for TTX-w, p = 0.24). (F) Schematic of stimulation paradigm to monitor the effect of protein synthesis. Translation elongation inhibitor (CHX, $50 \mathrm{mg} / \mathrm{ml})$ was added at 90 $\min (\mathrm{TTX}-\mathrm{w}+\mathrm{CHX})$. In another set of experiments, CHX was incubated for 1 hour and then washed out (TTX-w + CHX-w). (G) Representative images showing IE-transcription from both alleles, followed by shutdown maintained with $\mathrm{CHX}$ addition. Washout of CHX restored transcription. (H) Intensity trace of transcription sites from two conditions- with CHX addition (TTX-w + CHX), and washout (TTX-w + CHX-w). (I) Percentage of reactivation across conditions, each circle represents one experiment (TTX-w vs TTX-w + CHX, **** p < 0.001, TTX-w vs TTX-w + CHX washout, * p = 0.036, one-way ANOVA; $\mathrm{n}$ $=21$ neurons for TTX-w + CHX, $\mathrm{n}=47$ neurons for TTX-w + CHX-w, from 4 independent experiments, TTX-w from Fig 1E) (J) Frequency distribution of reactivation onset times after CHX washout. CV= coefficient of variation. Error bars indicate SEM. **** denotes $\mathrm{p}<0.001$, * denotes $\mathrm{p}<0.05$. Scale bar $5 \mu \mathrm{m}$.

Figure 4: Detection of Arc protein hubs in dendrites and mRNA localization to hubs. (A) Schematic of the Halo-Arc protein reporter driven by ESARE (activity-regulated promoter) to detect proteins from IE and second phase. (B) PCP-GFP construct to visualize endogenous Arc mRNAs. A cocktail of lentiviruses expressing A and B was used to image Arc proteins and mRNAs in the same neuron. (C) Schematic of JF646/JF549 labeling timeline after stimulation. Since transcriptional reactivation occurred at 150 min post stimulation, Arc mRNAs and proteins from the second phase were imaged $3 \mathrm{hr}$ onwards. (D-E) Representative image of dendritic Arc protein following JF646 labeling during the IE phase of 
protein synthesis. Differential intensity of JF646 signal shown in E. (F) Intensity profile of JF646 intensity showed distinct peaks in specific dendritic regions. (G) A $6 \mu \mathrm{m}$ segment around the local maxima in $\mathrm{F}$ was used to designate Arc protein hub from IE-stage (orange outline). A region of same dimension (dashed outline) nearby the hub was marked as neighboring site. (H) Labeling with JF549 shows Arc proteins synthesized in the second phase. Bottom panel shows a merged image of JF549 with JF646, indicating close proximity of both puncta. (I) Localization of endogenous Arc mRNAs in the same dendrite. Middle panel shows a kymograph, where localized mRNAs are numbered. (J) Normalized intensity trace of new Arc protein (JF549 signal) over time in hub versus neighboring site as defined in G. (K) Comparison of Arc mRNA counts populating the hubs versus neighboring sites $(* * * \mathrm{p}=0.002$, Wilcoxon signed rank test). (L) Comparison of the residence times of Arc mRNAs in the hubs wrt neighboring sites $(* \mathrm{p}=0.029) . \mathrm{n}=12$ neurons from 3 independent experiments $(\mathrm{J}, \mathrm{K}) . \mathrm{n}=43 \mathrm{mRNAs}$ for hub versus $n=23$ mRNAs for neighboring site (L). Scale bar is 6 microns. *** denotes $\mathrm{p}<0.005$, * denotes $\mathrm{p}<0.05$. Error bars represent SEM. Scale bar is 6 microns.

\section{Figure 5: Long-term imaging of Arc translation reveals hotspots and biphasic dynamics (A)}

Schematic of the Suntag-Arc translation reporter. The reporter is driven by ESARE (activity-regulated promoter) and contains the 24X GCN4 epitopes of Suntag upstream of the Arc CDS and followed by the 3' UTR of Arc, and stem loops MS2V7. (B) The translating mRNAs are detected by fixed-cell imaging with antibodies against GCN4 and by smFISH against GCN4 and stem loop sequence. In live cells, translation sites (TLS) are detected by binding of the single chain antibody against GCN4 (scFV) fused to superfolder GFP (sfGFP). (C) Images of dendrites showing both nascent peptides and mRNAs under stimulation conditions and inhibition with Harringtonine. Co-localization of IF-smFISH spots indicate translating mRNAs (yellow arrows). (D) Comparison of translating mRNAs after stimulation and translation inhibition (TTX-w $2 \mathrm{~h}$ vs TTX-w $2 \mathrm{~h}+$ Harringtonine, $* * * * \mathrm{p}<0.001$, TTXw $2 \mathrm{~h}$ vs TTXw 4h, $\mathrm{p}=0.23$, one-way ANOVA; $\mathrm{n}=49$ dendrites for TTX-w $2 \mathrm{~h}, \mathrm{n}=34$ dendrites for TTX-w $4 \mathrm{~h}, \mathrm{n}=37$ dendrites for TTX-w + Harringtonine from 2 independent experiments). (E) Stimulation and imaging paradigm to capture long-term dynamics of Arc TLS. (F) Images from a dendrite showing appearance of TLS (arrows). Different colors represent different TLS. Time was binned to represent IE (90-180 min) and second (181-270 $\mathrm{min}$ ) phase. Green dotted box indicates the ROI with TLS clustering, representing hotspots along the dendrite. (G) Counts of TLS show biphasic translation. (H) Inverse cumulative distribution function of OFF-periods fitted to a 2-component exponential, yielding two time constants $\tau 1$, $\tau 2$, and relative percentage of events indicated ( $\mathrm{n}=90$ events). (I) Schematic of translation hotspot, with clustering of at least 3 TLS in $8 \mu \mathrm{m}$ segment as the inclusion criterion. (J) Cumulative number of TLS in 
hotspots versus regions without hotspots ( $\mathrm{n}=20$ dendrites, 3 independent experiments). (K) Duration of translation bursts in hotspots during the IE and second phase ( $n=35$ events in IE, $n=22$ events in second phase). (L) Proposed model of emergence and maintenance of Arc protein hubs by local translation in the hotspots. Arc mRNAs from the IE-phase translated in specific hotspots promote rapid increase in nascent protein density to form the "hubs". During the OFF-phase, translation is low possibly due to limited mRNA availability. A second transcriptional cycle supplies mRNAs, which preferentially visit the hubs to undergo a second phase of translation, and consolidate the hub compared to the neighboring dendritic regions. Error bars indicate SEM. $* * * *$ denotes $\mathrm{p}<0.001$, * denotes $\mathrm{p}<0.05$, ns for $\mathrm{p}>0.05$. 
Figure 1
bioRxiv preprint doi: https://doi.org/10.1101/2021.10.29.466479; this version posted November 1, 2021. The copyright holder for this preprint (which was not certified by peer review) is the author/funder. All rights reserved. No reuse allowed without permission.

A TTX-washout
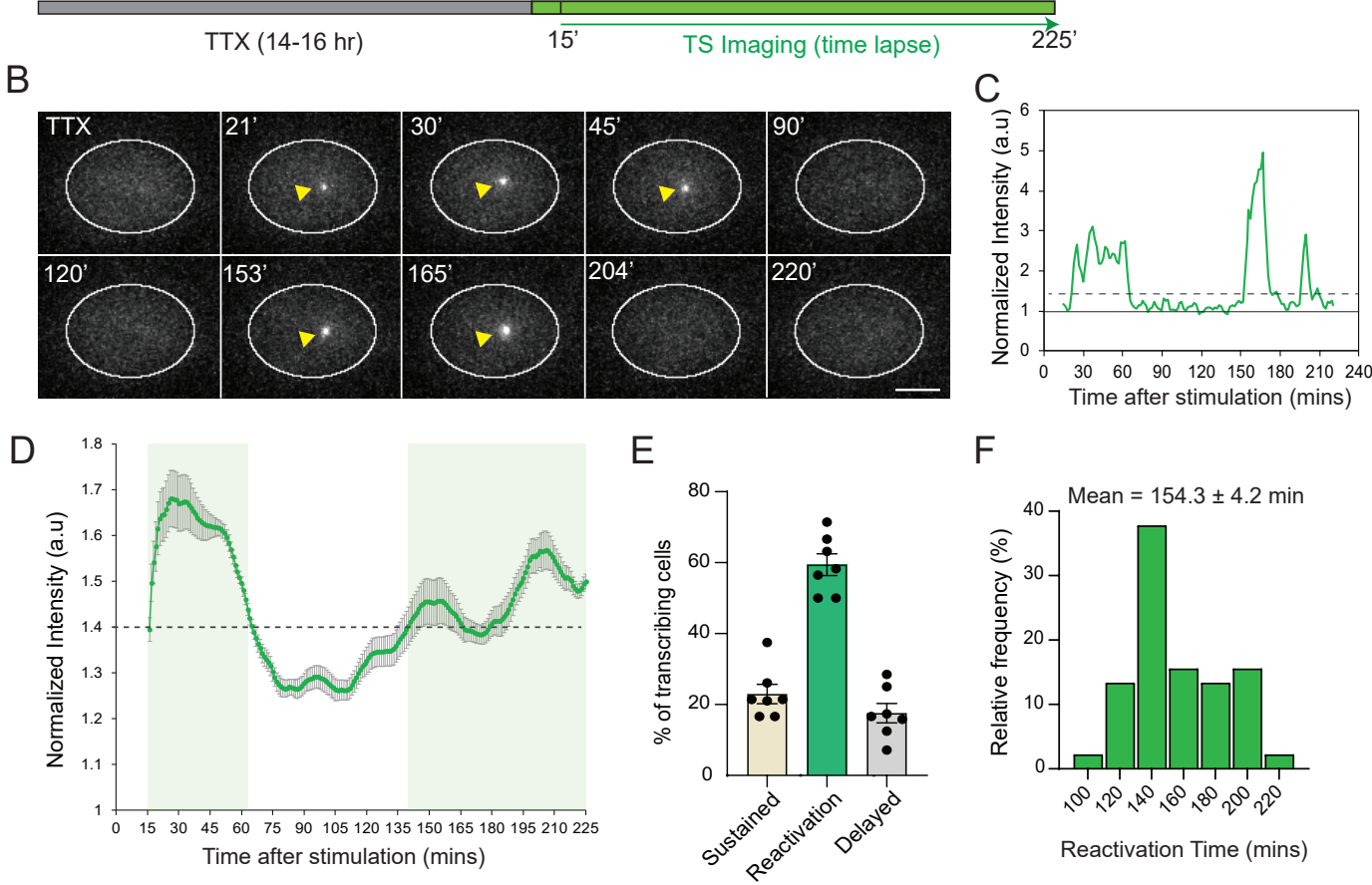

$\mathrm{F}$
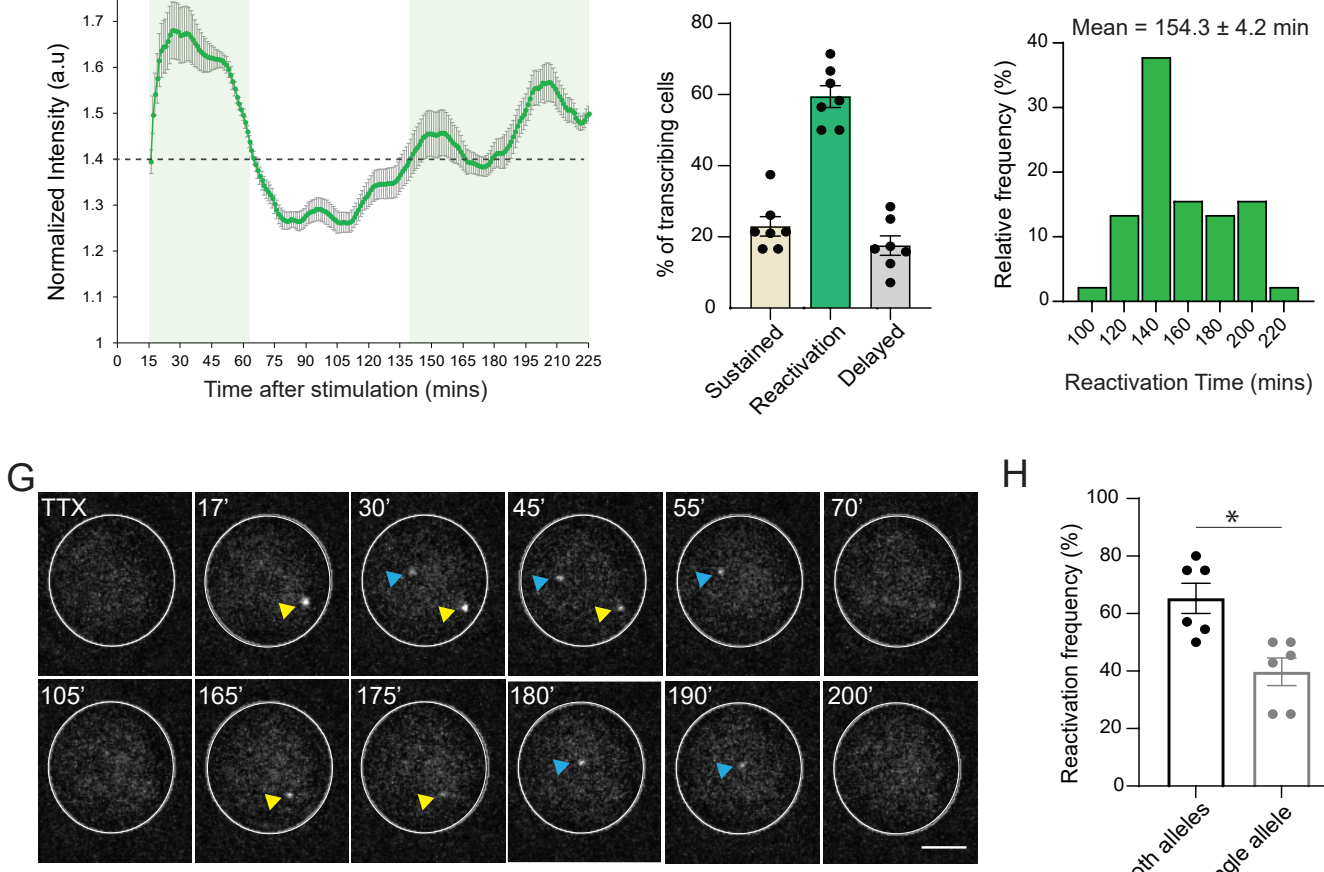

$\mathrm{H}$

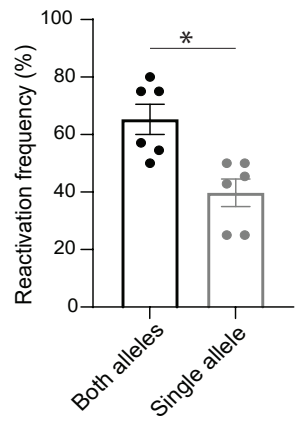


Figure 2

bioRxiv preprint doi: https://doi.org/10.1101/2021.10.29.466479; this version posted November 1, 2021. The copyright holder for this preprint (which was not certified by peer review) is the author/funder. All rights reserved. No reuse allowed without permission.

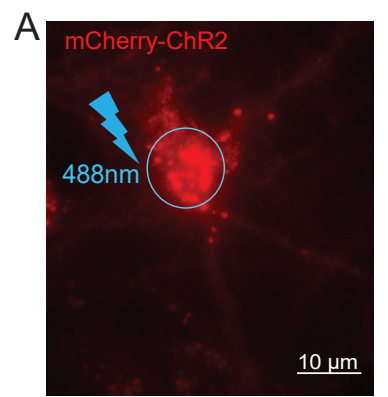

$$
\text { B } \begin{gathered}
488 \mathrm{~nm} \text { trains } \\
(25 \text { pulses @20 Hz) }
\end{gathered}
$$

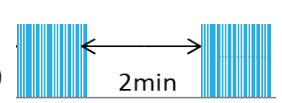

E F
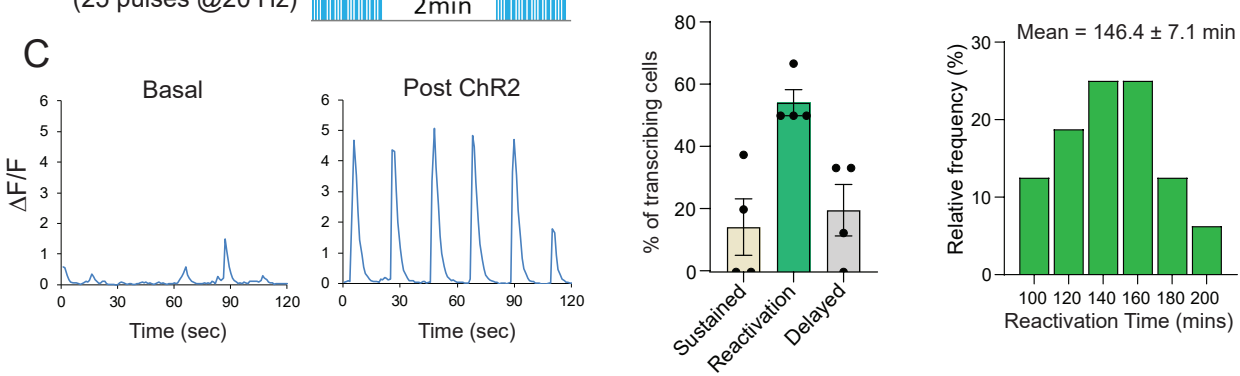

D
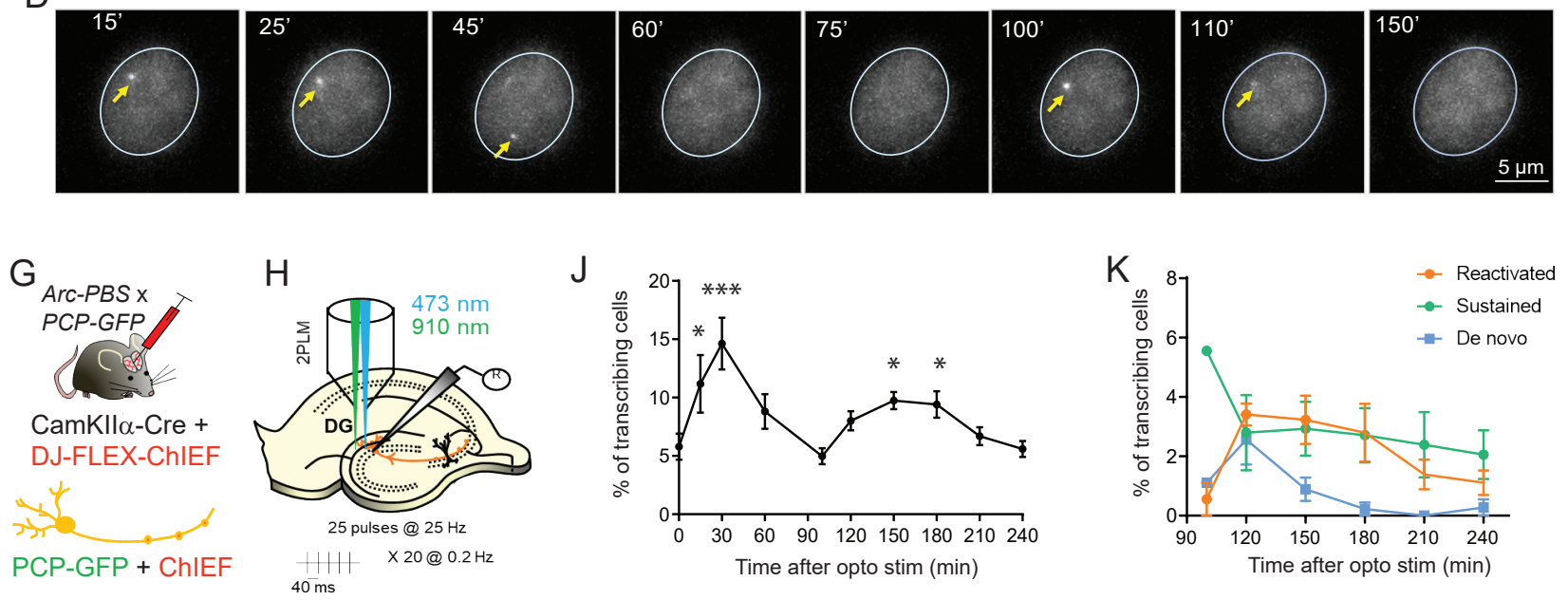

I

After optical stimulation of GCs in hippocampal slices
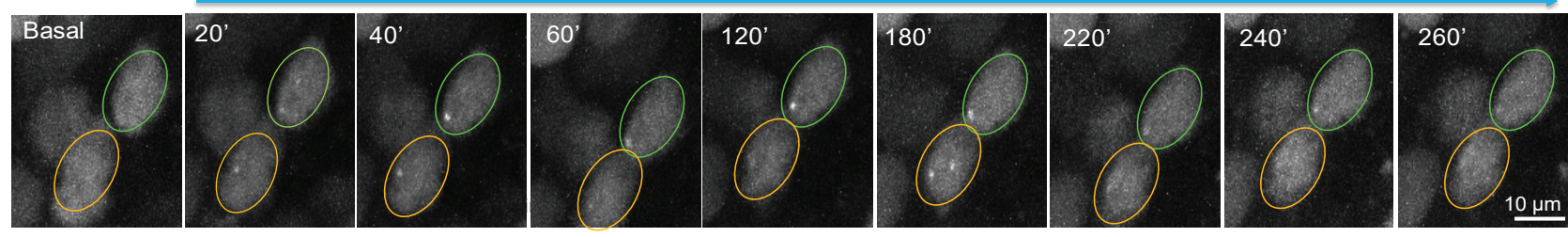
Figure 3

bioRxiv preprint doi: https://doi.org/10.1101/2021.10.29.466479; this version posted November 1, 2021. The copyright holder for this preprint (which was not certified by peer review) is the author/funder. All rights reserved. No reuse allowed without permission.

A

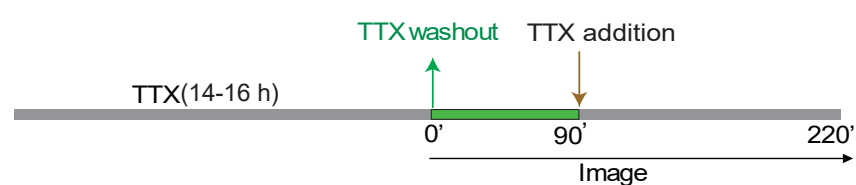

B Nuclear $\mathrm{Ca} 2+$

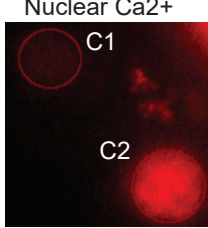

C
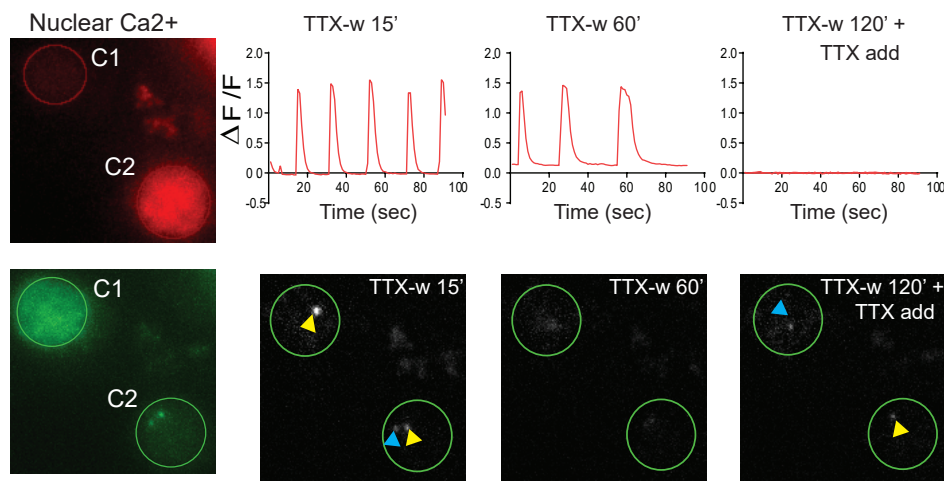

TTXwashout

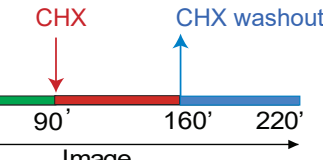

\section{G}

GTX-w

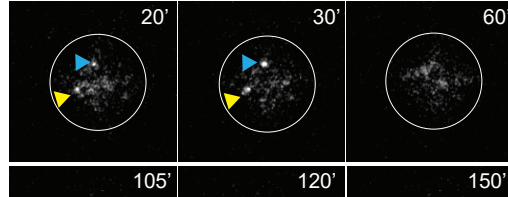

TTX-w+

$\mathrm{CHX}$
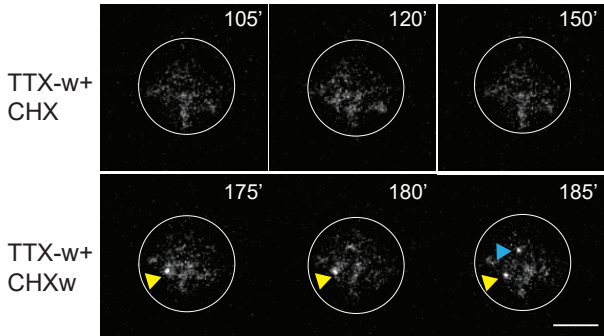
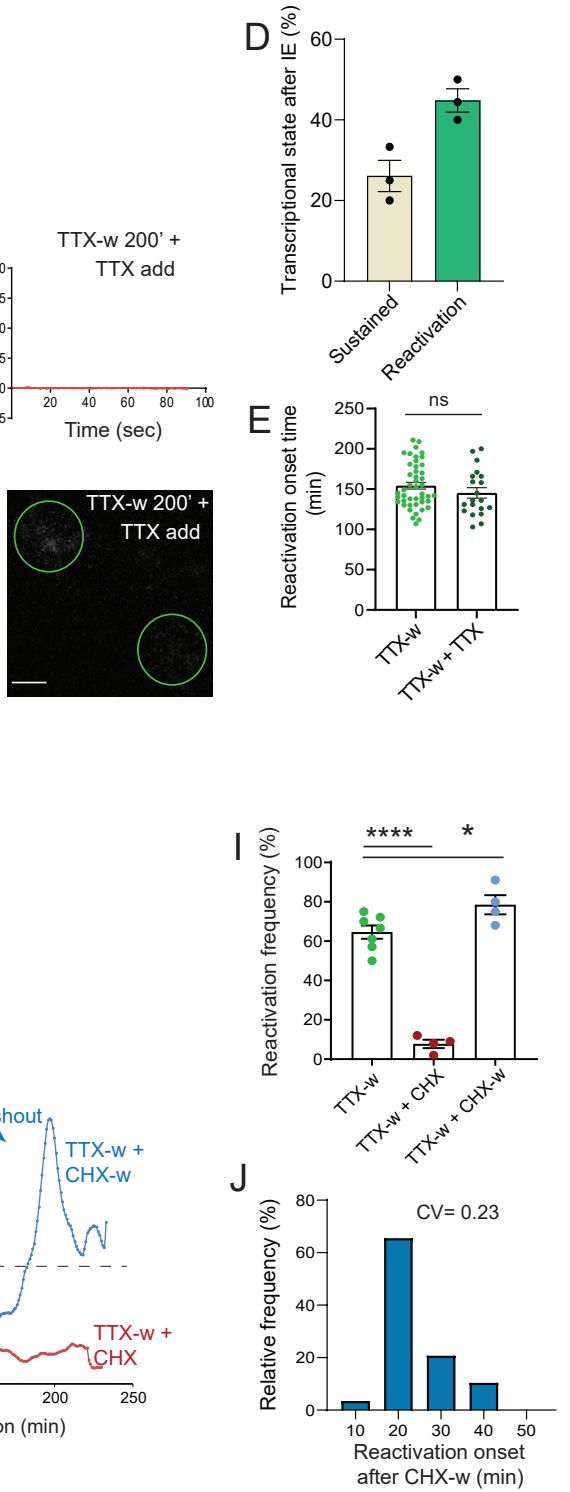
Figure 4

bioRxiv preprint doi: https://doi.org/10.1101/2021.10.29.466479; this version posted Nevember 1, 2021. The copyright holder for this preprint (whichAvas not certified by peer review) is the author/funder. All rightBre\$erved. No reuse allowed without permission. \begin{tabular}{|l|l|l|l|l|l|l|l|l|l|}
\hline ESARE & Halo & Arc-CDS & Arc 3'UTR \\
\hline
\end{tabular}

(detect Arc proteins from IE and 2nd phase)

(detect PP7-tagged Arc mRNAs)
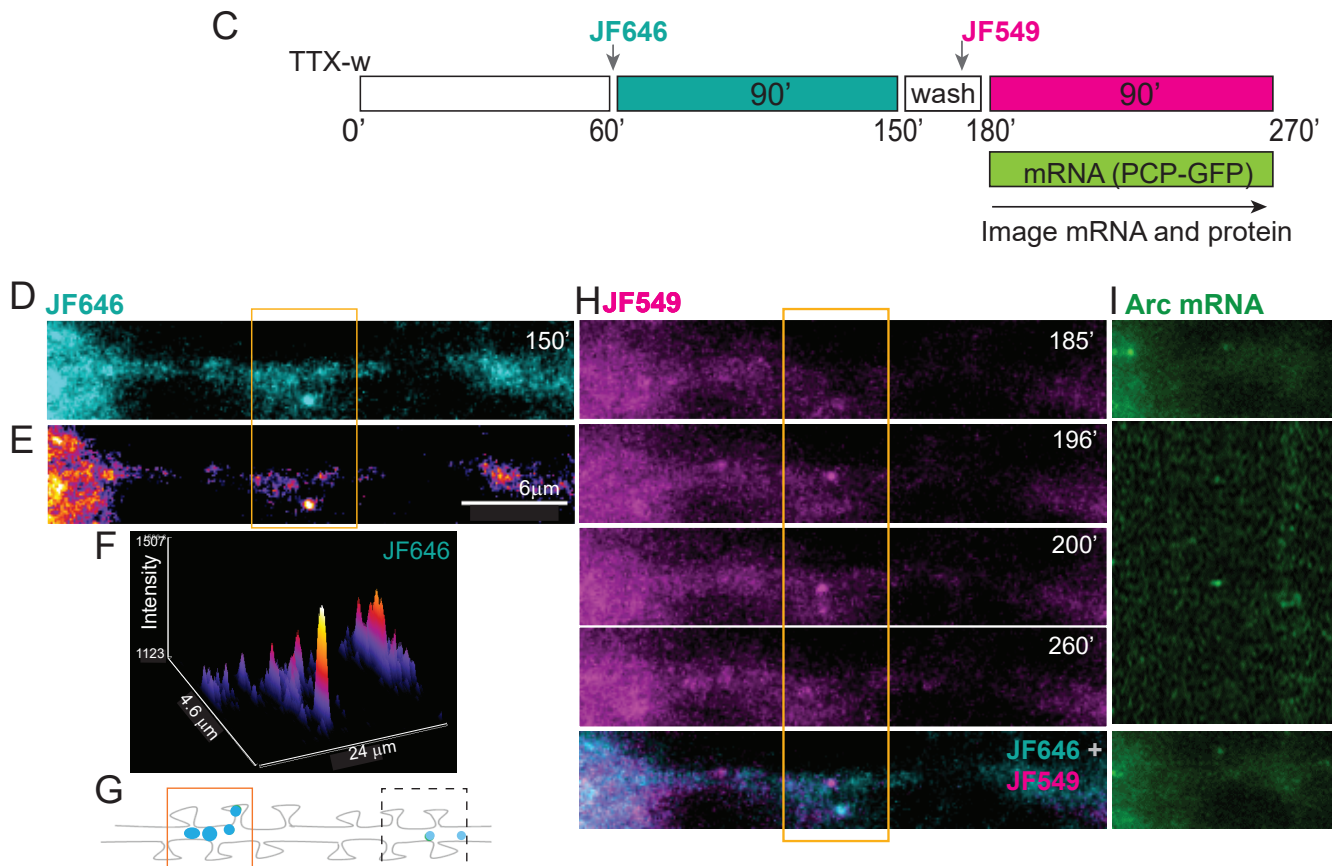

Arc protein $\mathrm{Hub}$ (IE-phase)

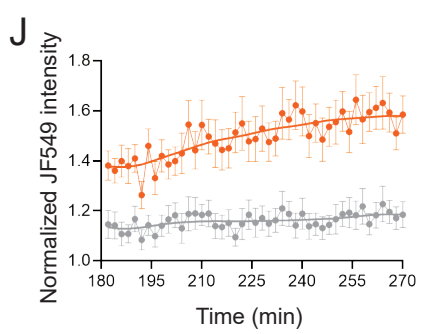

- Arc protein Hub Neighboring site
K

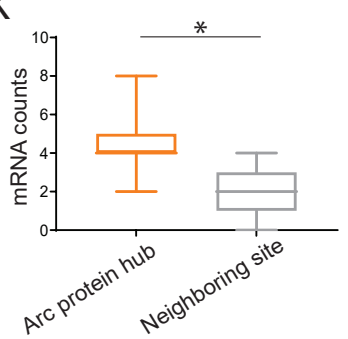

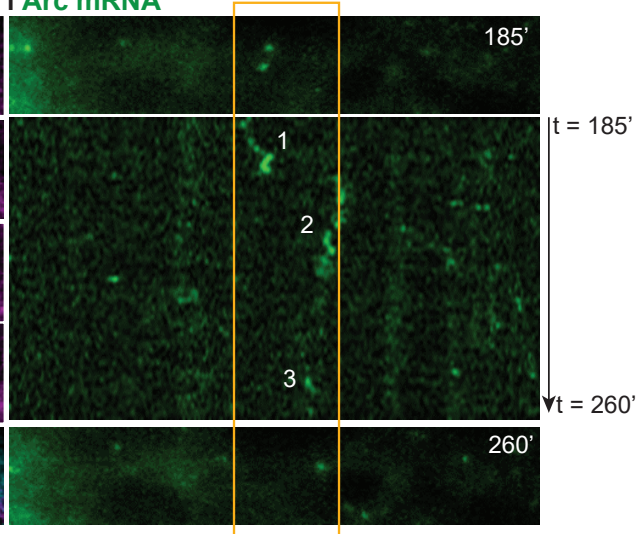

L

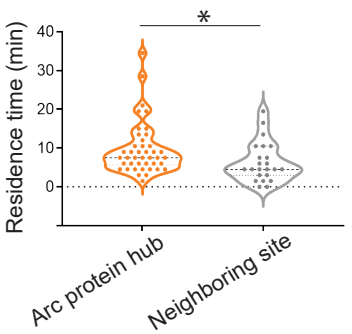


Figure 5

bioRxiv preprint doi: https://doi.org/10.1101/2021.10.29.466479; this version posted November 1, 2021. The copyright holder for this preprint (which was not certified by peer review) is the author/funder. All rights reserved. No reuse allowed without permission.
A
\begin{tabular}{|l|l|l|l|l|}
\hline & \\
\hline ESARE & $24 \times$ GCN4 & Arc-CDS & Arc-3'UTR & MS2V7 \\
\hline
\end{tabular}
B

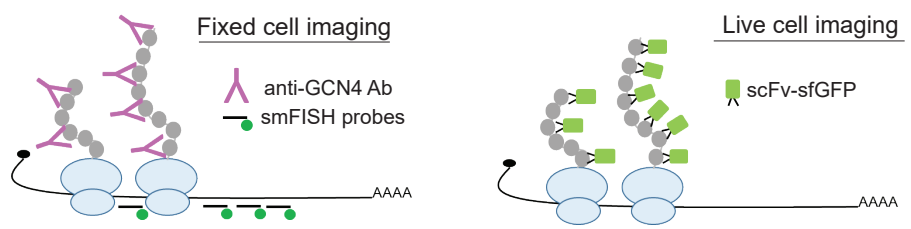

C

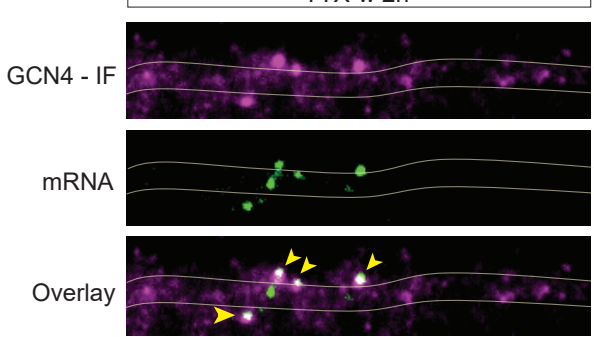

TTX-w 2h + Harringtonine

D
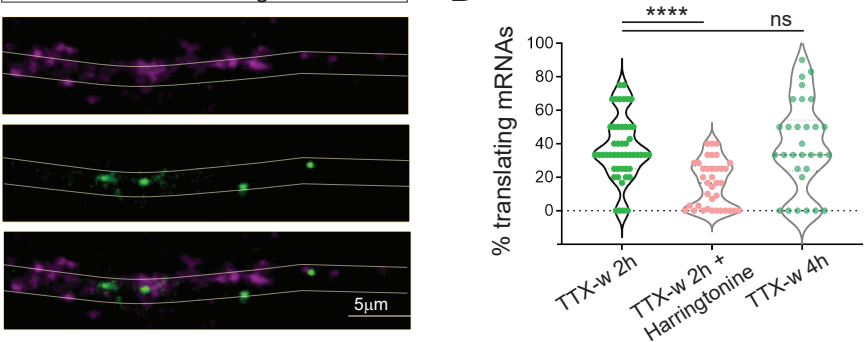

E

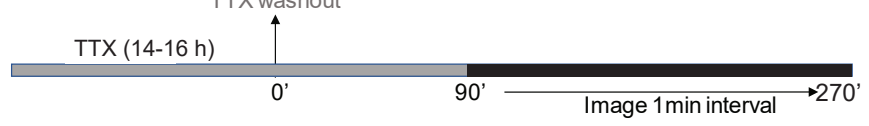

F

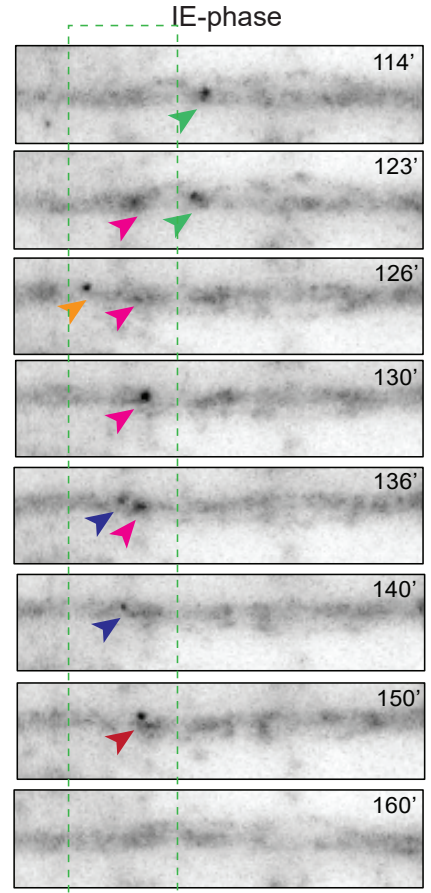

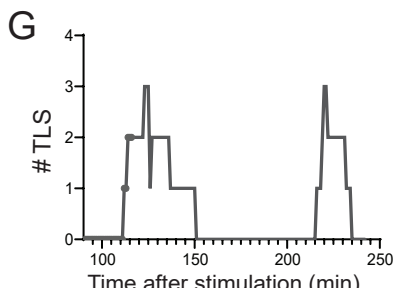
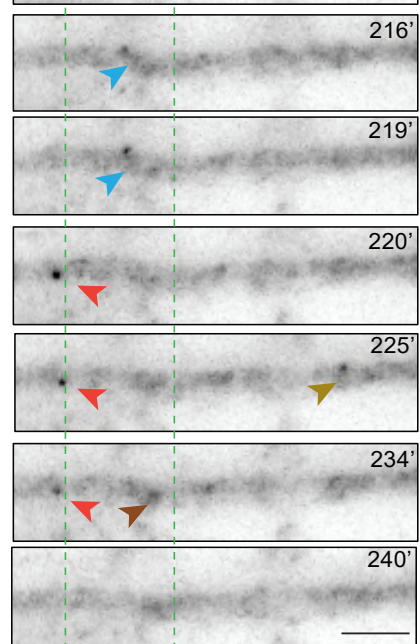

Time after stimulation (min)

I

$\mathrm{H} \quad \tau 1=7.1(25 \%)$

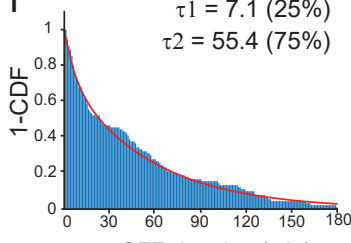

OFF-duration (min)

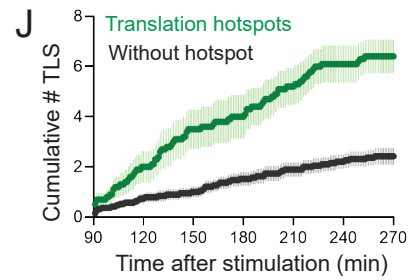

K

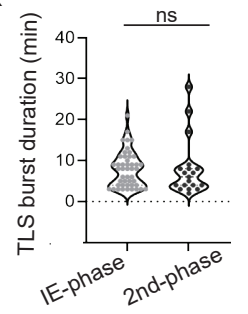

L Translation hotspot without hotspot - arctls r al r r s r ie- nascent s is is 25 is phase Arc protein

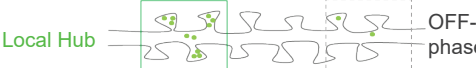

Consolidating $\quad \Omega \quad \Omega \Omega S \Omega$ 2ndthe hub 25.52525258 phase 